\title{
Hepatic transcriptome implications for palm fruit juice deterrence of type 2 diabetes mellitus in young male Nile rats
}

\author{
Soon-Sen Leow ${ }^{1 *}$ D, Julia Bolsinger ${ }^{2}$, Andrzej Pronczuk ${ }^{2}$, K. C. Hayes ${ }^{2}$ and Ravigadevi Sambanthamurthi ${ }^{1 *}$
}

\begin{abstract}
Background: The Nile rat (NR, Arvicanthis niloticus) is a model of carbohydrate-induced type 2 diabetes mellitus (T2DM) and the metabolic syndrome. A previous study found that palm fruit juice (PFJ) delayed or prevented diabetes and in some cases even reversed its early stages in young NRs. However, the molecular mechanisms by which PFJ exerts these anti-diabetic effects are unknown. In this study, the transcriptomic effects of PFJ were studied in young male NRs, using microarray gene expression analysis.

Methods: Three-week-old weanling NRs were fed either a high-carbohydrate diet (\%En from carbohydrate/fat/ protein $=70: 10: 20,16.7 \mathrm{~kJ} / \mathrm{g} ; n=8$ ) or the same high-carbohydrate diet supplemented with PFJ (415 $\mathrm{ml}$ of 13,000-ppm gallic acid equivalent (GAE) for a final concentration of $5.4 \mathrm{~g}$ GAE per kg diet or $2.7 \mathrm{~g}$ per $2000 \mathrm{kcal}$; $n=8$ ). Livers were obtained from these NRs for microarray gene expression analysis using Illumina MouseRef-8 Version 2 Expression BeadChips. Microarray data were analysed along with the physiological parameters of diabetes.

Results: Compared to the control group, 71 genes were up-regulated while 108 were down-regulated in the group supplemented with PFJ. Among hepatic genes up-regulated were apolipoproteins related to high-density lipoproteins (HDL) and genes involved in hepatic detoxification, while those down-regulated were related to insulin signalling and fibrosis.

Conclusion: The results obtained suggest that the anti-diabetic effects of PFJ may be due to mechanisms other than an increase in insulin secretion.
\end{abstract}

Keywords: Palm fruit juice, Oil palm phenolics, Antioxidants, Diabetes, Metabolic syndrome, Gene expression, Nile rat

\section{Background}

Nutritional overload and sedentary lifestyle give rise to the prevalence of type 2 diabetes mellitus (T2DM) in modern societies, and this chronic disease is estimated to reach 439 million cases by 2030 [87]. Although T2DM is a disease of adults, it is an increasingly common diagnosis among adolescents in high-risk countries such as Asia, the Middle East, and the USA [46]. T2DM is characterised by insulin resistance, declining insulin production and eventual pancreatic $\beta$ cell failure [71]. This leads to a decrease in glucose transport into liver, muscle and fat cells and an increase in circulating

\footnotetext{
*Correspondence: ssleow@mpob.gov.my; raviga@mpob.gov.my

${ }^{1}$ Malaysian Palm Oil Board, No. 6, Persiaran Institusi, Bandar Baru Bangi, 43000

Kajang, Selangor, Malaysia

Full list of author information is available at the end of the article
}

glucose. T2DM is often associated with increasing obesity, via a combination of clinical abnormalities known as the metabolic syndrome, which comprises insulin resistance, visceral adiposity, hypertension, atherogenic dyslipaemia and endothelial dysfunction [32]. These conditions are interrelated and share common mediators, pathways and pathophysiological mechanisms [50]. The metabolic syndrome is a state of chronic low-grade inflammation linked to aberrant energy metabolism as a consequence of complex interplay between genetic and environmental factors [57].

Due to the growing concern over T2DM and the metabolic syndrome, animal models that mimic these human diseases are needed to assess possible antidiabetic preventative or therapeutic measures [128]. The Nile rat (NR), also known as the African grass rat 
(Arvicanthis niloticus), has been described as a relevant model of T2DM and the metabolic syndrome, as it allows for detailed nutritional modelling of diet-induced T2DM similar to that in humans. The NR spontaneously develops hyperinsulinaemia, hyperglycaemia with dyslipaemia and hypertension in the early phase of the disease $[14,16,21,85]$. Further characterisation revealed that NRs develop liver steatosis, abdominal fat accumulation, nephropathy, atrophy of pancreatic islets of Langerhans and fatty streaks in the aorta, as well as hypertension [14, 16, 21, 85]. Males are more prone than females, with rapid progression to T2DM depending on the glycaemic load of the challenge diet and cumulative glycaemic load [15]. Although diet challenge appears as the primary factor and dietary intervention can modulate the development of T2DM and metabolic syndrome in NRs, genetic susceptibility also plays a pivotal role, similar to humans. This rodent model thus represents a novel system of gene-diet interactions affecting energy utilisation that can provide insights into the prevention and treatment of diabetes, as well as the metabolic syndrome [14, 21]. As in humans, the NR is sensitive to the daily glycaemic load and as such reliably mirrors the course of T2DM and the metabolic syndrome observed in humans [14].

At present, no cure has been found for T2DM and the metabolic syndrome. Treatment methods normally suggested include lifestyle modifications, treatment of obesity that induces weight reduction, oral anti-diabetic medication that reduces intestinal glucose absorption, increases insulin sensitivity or exerts insulin-sensitising effects or lastly insulin injections [87]. All the above measures have been shown to prevent T2DM in the NR. However, current research strongly supports the concept that the consumption of certain fruits and plant-derived foods is inversely correlated with prevalence of T2DM and the metabolic syndrome $[8,35,80]$. A great array of phenolic compounds may exert anti-diabetic effects either directly or indirectly [1]. Phenolic compounds may influence glucose metabolism by several mechanisms, such as inhibition of carbohydrate digestion and glucose absorption in the small intestine, stimulation of insulin secretion from pancreatic $\beta$ cells, modulation of hepatic gluconeogenesis, activation of insulin receptors and glucose uptake in insulin-sensitive tissues (thus enhancing insulin sensitivity) and modulation of gut flora activity, as well as modulation of intracellular signalling pathways and gene expression influencing glucose utilisation [26, 47, 79]. Some examples of plant phenolic compounds which were found to display anti-diabetic effects in humans include resveratrol [82, 110], olive leaf extracts [28, 125], pomegranate juice [88] and green tea extracts [61,69].

The oil palm (Elaeis guineensis) fruit contains phenolic compounds [99], which are extracted from the aqueous vegetation liquor produced during oil palm milling. Palm fruit juice (PFJ) consists mainly of phenolic acids, including three caffeoylshikimic acid isomers and $p$-hydroxybenzoic acid [99]. PFJ has been shown to display antioxidant properties and confer positive outcomes on degenerative diseases in various animal models without evidence of toxicity $[16,22,65-68,99,100,103]$. In relation to T2DM, we have previously shown that PFJ blocked T2DM progression in 12-week-old male NRs, with a substantial decrease in blood glucose after 17 weeks of treatment [100]. In addition, PFJ delayed T2DM onset or completely prevented it during the intervention period and even reversed advancing T2DM in young NRs [16]. PFJ has also been shown to deter T2DM complications, including retinopathy and nephropathy in NRs [14, 21, 85], not unlike other plant polyphenols [5]. PFJ thus has demonstrated anti-diabetic effects. However, the detailed molecular mechanisms by which PFJ effects these changes in NRs have yet to be explored, prompting the microarray gene expression analysis in the present study.

\section{Methods \\ Animal feeding and sample collection}

Three-week-old male NRs $(n=16)$ were divided into two groups, controls without PFJ $(n=8)$ and PFJ $(n=8)$. We chose to study 3-week-old Nile rats for 4 weeks in this study as this window of development is the most sensitive to the development of nutritionally induced T2DM in the NR and thus provides the highest chances of altering this development through the application of PFJ. This would help pinpoint the gene expression changes caused by PFJ in deterring the occurrence of diabetes more efficiently $[16,21]$. Early diabetes (7 weeks of age) in Nile rats is detected by random blood glucose levels, whereas diabetic fasting blood glucose does not always manifest until 12 weeks of age [85]. In addition, only males were used in this experiment as they develop T2DM more readily than females, presumably based on sex hormone differences [21]. NRs in the control group were fed a semi-purified high-carbohydrate diet ad libitum (\% En from carbohydrate $/$ fat $/$ protein $=70: 10: 20,16.7 \mathrm{~kJ} / \mathrm{g}$ ), while those in the $\mathrm{PFJ}$ group were given liquid PFJ incorporated directly into the same diet ( $415 \mathrm{ml}$ of 13,000 ppm gallic acid equivalent (GAE) for a final concentration of 5.4 g GAE per kg diet or $2.7 \mathrm{~g}$ per $2000 \mathrm{kcal}$ (daily human equivalent)) (Table 1 ). The composition of PFJ was as described previously [99], with major phenolic components being three caffeoylshikimic acid isomers and p-hydroxybenzoic acid. Body weight was assessed throughout the feeding period, as were food (in $\mathrm{g} / \mathrm{d}$ and $\mathrm{kJ} / \mathrm{d}$ ) and fluid intakes. After 4 weeks, random and fasting blood glucose levels were assessed, along with terminal organ weights, plasma lipids and insulin. All experiments and procedures were approved by the Brandeis University Institutional Animal Care and Use Committee. 


\section{Food efficiency}

Food efficiency was calculated by dividing body weight gain (in $\mathrm{g} / \mathrm{d}$ ) by caloric intake (in $\mathrm{kJ} / \mathrm{d}$ ) and multiplying the result by 1000 . Results represent the body weight gained (g) per $1000 \mathrm{~kJ}$ consumed.

\section{Random and fasting blood glucose}

Blood glucose was measured in $\mathrm{O}_{2} / \mathrm{CO}_{2}$-anaesthetised NRs from a drop of tail blood, obtained by lancet puncture of the lateral tail vein using an Elite XL glucometer (Bayer Co., Elhart, IN). Random blood glucose (RBG) was assessed in non-fasted NRs between 9 and 10 am on non-feeding days (semi-purified diets were replenished three times per week). Fasting blood glucose (FBG) was measured at about 9 to $10 \mathrm{am}$ after $16 \mathrm{~h}$ of overnight food deprivation.

\section{Terminal organ weights}

Organs (livers, kidneys, caecum and adipose) were weighed after excision, and their weights (in g) were divided by the terminal body weight (in g) to obtain a percentage. The livers were snap-frozen in liquid nitrogen and stored at $-80{ }^{\circ} \mathrm{C}$ until the total RNA extraction process for gene expression analysis. The relative carcass weight (as percentage body mass) was determined by weighing lean body mass (after exsanguination and excision of all organs) and dividing it by the terminal body weight (in g). Carcass weight was included as an indicator of muscle growth. Body length (nose to base of tail, in $\mathrm{cm}$ ) was also included as a parameter of growth.

\section{Plasma biochemical measurements}

Plasma triacylglycerol (TG) and total cholesterol (TC) were determined spectrophotometrically using Infinity ${ }^{\mathrm{TM}}$ kits (Thermo Fisher Scientific Inc., Middletown, VA, TG ref \# TR22421, TC ref \# TR13421). Plasma insulin was determined with an ELISA kit for rat/mouse insulin (Linco Research, EMD Millipore, Billerica, MA, ref \# EZRMI-13K), according to the manufacturer's protocol.

\section{Statistical analyses}

Statistical analyses on physiological and biochemical parameters were performed using the Super ANOVA statistical software (Abacus Concepts, Inc., Berkeley, CA). Two-tailed unpaired Student's $t$ test was performed, and differences with $p$ values of less than 0.05 were considered statistically significant.

\section{Microarray gene expression analysis}

Total RNA isolation from frozen NR livers was conducted using the RNeasy Plus Mini Kit (Qiagen, Inc., Valencia, CA) and QIAshredder homogenisers (Qiagen, Inc., Valencia, CA), preceded by grinding in liquid nitrogen using mortars and pestles. The total RNA samples obtained were subjected to NanoDrop 1000A Spectrophotometer (Thermo Fisher
Table 1 Composition of high-carbohydrate diet

\begin{tabular}{ll}
\hline Component & Amount $(\mathrm{g} / \mathrm{kg})$ \\
\hline En & 70 \\
Carbohydrate & 10 \\
Fat & 20 \\
Protein & 16.7 \\
En (kJ/g) & \\
Ingredients (g/kg) & 100 \\
Casein & 100 \\
Lactalbumin & 350 \\
Dextrose & $288(+60$ with gel) \\
Corn starch & 44 \\
Fat & 8 \\
Butter (g of fat) & 15 \\
Tallow & 23 \\
Soybean oil & 44 \\
Mineral mix & 11 \\
Vitamin mix & 3 \\
Choline chloride & 0.6 \\
Cholesterol &
\end{tabular}

${ }^{\mathrm{a}} 60 \mathrm{~g}$ corn starch was added to $800 \mathrm{ml}$ water to form a gel or added to $375 \mathrm{ml}$ water $+415 \mathrm{ml}$ PFJ (13,000 ppm GAE for a final concentration of $5.4 \mathrm{~g}$ GAE per kg diet or $2.7 \mathrm{~g}$ per $2000 \mathrm{kcal})$

${ }^{\mathrm{b}}$ Ausman-Hayes salt mix. Mineral mix contained the following ( $\mathrm{g} / \mathrm{kg} \mathrm{mix}$ ): magnesium oxide, 320; calcium carbonate, 290.5; potassium phosphate dibasic, 312.2; calcium phosphate dibasic, 72.6; magnesium sulphate, 98.7; sodium chloride, 162.4 ; ferric citrate, 26.6 ; potassium iodide, 0.77 ; manganese sulphate, 3.66 ; zinc chloride, 0.24 ; cupric sulphate, 0.29 ; chromium acetate, 0.044 ; sodium selenite, 0.004

CHayes-Cathcart vitamin mix. Vitamin mix contained the following ( $\mathrm{g} / \mathrm{kg} \mathrm{mix}$ ): D-a-tocopheryl acetate (500 IU/g), 15; inositol, 5; niacin, 3; calcium pantothenate, 1.6; retinyl palmitate $(500,000 \mathrm{IU} / \mathrm{g}), 1.5$; cholecalciferol $(400,000 \mathrm{IU} / \mathrm{g}), 0.1$; menadione, 0.2 ; biotin, 0.02 ; folic acid, 0.2 ; riboflavin, 0.7 ; thiamin, 0.6; pyridoxine $\mathrm{HCl}, 0.7$; cyanocobalamin, 0.001; dextrin, 972

Scientific, Waltham, MA) measurement for yield and purity assessment. Integrity of the total RNA samples was then assessed using the Agilent 2100 Bioanalyzer (Agilent Technologies, Santa Clara, CA) and Agilent RNA 6000 Nano Chip Assay Kit (Agilent Technologies, Santa Clara, CA).

Amplification of total RNA samples which were of high yield, purity and integrity was performed using the Illumina TotalPrep RNA Amplification Kit (Ambion, Inc., Austin, TX). The complementary ribonucleic acid (cRNA) produced was then hybridised to the Illumina MouseRef-8 Version 2 Expression BeadChip (Illumina, Inc., San Diego, CA), using the Direct Hybridization Kit (Illumina, Inc., San Diego, CA). As each MouseRef-8 BeadChip enables the interrogation of eight samples in parallel, a total of eight cRNA samples were used in the microarray experiment, by selecting four total RNA samples with the highest RNA integrity numbers and 28S/ $18 \mathrm{~S}$ ribosomal RNA (rRNA) ratios within each condition. Microarray hybridisation, washing and scanning were conducted according to the manufacturer's 
instructions. The raw gene expression data obtained are available at Gene Expression Omnibus [33] (accession number: GSE64901).

Quality control of the hybridisation, microarray raw data extraction and initial analysis were performed using the Illumina BeadStudio software (Illumina, San Diego, CA). Outlier samples were also removed via hierarchical clustering analysis provided by the Illumina GenomeStudio software, via different distance metrics including correlation, absolute correlation, Manhattan and Euclidean distance metrics. Gene expression values were normalised using the rank invariant method, and genes which had a detection level of more than 0.99 in either the control or treatment samples were considered significantly detected.

To filter the data for genes which changed significantly in terms of statistics, the Illumina Custom error model was used and genes were considered significantly changed at a differential score of more than 13, which was equivalent to a $p$ value $<0.05$. Two-way (gene and sample) hierarchical clustering of the significant genes was then performed using the TIGR MeV software to ensure that the replicates of each condition were clustered to each other. The Euclidean distance metric and average linkage method were used to carry out the hierarchical clustering analysis. The genes and their corresponding data were then exported into the Microsoft Excel software for further analysis. To calculate fold changes, an arbitrary value of 10 was given to expression values which were less than 10 . Fold changes were then calculated by dividing the mean values of signal Y (treatment) with those of signal $\mathrm{X}$ (control) if the genes were upregulated and vice versa if the genes were downregulated.

Changes in biological pathways and gene ontologies (biological processes) were then assessed via functional enrichment analysis, using the GO-Elite software. The GO-Elite software ranks WikiPathways [58, 92] and gene ontologies based on the hypergeometric distribution. WikiPathways and gene ontologies which had permuted $p$ values of less than 0.05 , numbers of genes changed of more than or equal to 2 and $Z$ scores of more than 2 were considered significantly changed. In this study, upregulated and down-regulated genes were analysed separately in the functional enrichment analysis but were viewed together in each WikiPathway, using the PathVisio software [122]. For each of these WikiPathways, boxes coloured yellow indicate genes which were upregulated while those coloured blue indicate genes which were down-regulated. The fold changes of the genes were indicated next to their boxes.

Changes in regulatory networks were also analysed through the use of the Ingenuity Pathways Analysis software (Ingenuity ${ }^{\circ}$ Systems, Redwood City, CA). A network is a graphical representation of the molecular relationships between genes or gene products. Genes or gene products were represented as nodes, and the biological relationship between two nodes was represented as an edge (line). The intensity of the node colour indicates the degree of up-regulation (red) or downregulation (green). Nodes were displayed using various shapes that represented the functional class of the gene product. Edges were displayed with various labels that described the nature of the relationship between the nodes.

\section{Real-time qRT-PCR validation}

Two-step real-time quantitative reverse transcriptionpolymerase chain reaction (qRT-PCR) was conducted using TaqMan Gene Expression Assays (Applied Biosystems, Foster City, CA) to validate the microarray data obtained. This was performed on six differentially expressed target genes of interest (Table 2), which were selected based on the microarray data analysis performed. The same aliquots of total RNA samples used in the microarray experiments were utilised for this analysis. Primer and probe sets for the selected genes were obtained from the ABI Inventoried Assays-On-Demand (Applied Biosystems, Foster City, CA).

Briefly, reverse transcription to generate first-strand complementary deoxyribonucleic acid (cDNA) from total RNA was conducted using the High-Capacity cDNA Reverse Transcription Kit (Applied Biosystems, Foster City, CA). Real-time PCR was then performed on the first-strand cDNA generated using a $25 \mu \mathrm{L}$ reaction volume in an Applied Biosystems 7000 Real-Time PCR System (Applied Biosystems, Foster City, CA) using the following conditions: $50{ }^{\circ} \mathrm{C}, 2 \mathrm{~min}, 1$ cycle; $95{ }^{\circ} \mathrm{C}$, 10 min, 1 cycle; $95{ }^{\circ} \mathrm{C}, 15 \mathrm{~s}$ and $60^{\circ} \mathrm{C}, 1 \mathrm{~min}, 40$ cycles. For gene expression measurements, reactions for each biological replicate and non-template control (NTC) were performed in duplicates. For amplification efficiency determination, reactions were performed in triplicates.

Quality control of the replicates used, real-time qRTPCR data extraction and initial analysis were conducted using the 7000 Sequence Detection System software (Applied Biosystems, Foster City, CA). A manual threshold of 0.6000 and an auto baseline were applied in order to obtain the threshold cycle $(\mathrm{Ct})$ for each measurement taken. The threshold was chosen as it intersected the exponential phase of the amplification plots [19]. The criteria for quality control of the data obtained include $\Delta \mathrm{Ct}$ less than 0.5 between technical replicates and $\Delta \mathrm{Ct}$ more than 5.0 between samples and NTCs [86].

Relative quantification of the target genes of interest was performed using the qBase 1.3.5 software (Center for Medical Genetics, Ghent University Hospital, Ghent, 
Table 2 Genes selected for the real-time qRT-PCR validation experiment

\begin{tabular}{llll}
\hline Symbol & Definition & Accession & Assay ID \\
\hline Target genes & & NM_007469 & Mm00431816_m1 \\
Apoc1 & Apolipoprotein C-I & NM_023114 & Mm00445670_m1 \\
Apoc3 & Apolipoprotein C-III & NM_022012 & Mm00491529_m1 \\
Map3k11 & Mitogen-activated protein kinase kinase kinase 11 & NM_011946 & Mm00442451_m1 \\
Map3k2 & Mitogen-activated protein kinase kinase kinase 2 & NM_181585 & Mm00725026_m1 \\
Pik3r3 & Phosphatidylinositol 3-kinase, regulatory subunit, polypeptide 3 (p55) & NM_011503 & Mm00441589_m1 \\
Stxbp2 & Syntaxin binding protein 2 & & \\
Reference genes & & NM_009838 & Mm00486818_m1 \\
Cct6a & Chaperonin containing Tcp1, subunit 6a (zeta) & NM_008277 & Mm00801734_m1 \\
Hpd & 4-hydroxyphenylpyruvic acid dioxygenase & NM_027707 & Mm01297452_m1 \\
Nipbl & Nipped-B homologue (Drosophila) & NM_178281 & Mm01273530_m1 \\
Trim39 & Tripartite motif-containing 39 & & \\
\hline
\end{tabular}

The six target genes were selected based on their functional significance, their statistical significance, their presence as single splice transcripts in microarrays and their availability as Taqman assays designed across splice junctions. From the microarray data obtained, four candidate reference genes were also chosen to be tested for expression stability across the groups, with the three most stable ones being finally selected for relative quantification of the target genes

Belgium) [48], which takes into account the calculations of amplification efficiencies and multiple housekeeping genes. Expression levels of target genes were normalised to the geometric mean of the three most stable reference genes, selected out of the four tested (Table 2). Stability of these reference genes was assessed using the geNorm 3.5 software (Center for Medical Genetics, Ghent University Hospital, Ghent, Belgium) [123].

\section{Results}

\section{Physiological and biochemical parameters}

NRs fed the PFJ-supplemented diet consumed about $15 \%$ fewer calories $(p<0.05)$ than control rats and were associated with significantly lower body weights $(p<$ 0.05) (Table 3). Fluid intake did not significantly differ between the two groups. NRs in the PFJ group had less adipose tissue $(p<0.05)$ and a tendency for greater carcass weight (an indicator of lean body mass) and food efficiency. Their caeca were heavier too $(p<0.05)$ compared to the control group. NRs in the PFJ group had significantly lower levels of RBG $(p<0.05)$ and plasma TG $(p<0.05)$ compared to the control group, whereas no significant differences in overnight FBG were observed. Although TC in the PFJ group was slightly greater than that in the control group, it was not significant $(p>0.05)$. Insulin levels also did not differ between the two groups. Liver and kidney weights as percentages of body weights were similar between groups.

\section{Microarray gene expression}

Analysis of microarray gene expression of the NR livers revealed that 71 genes were up-regulated, while 108 genes were down-regulated in the PFJ group compared to the control group (Table 4). A few apolipoprotein genes, including Apoa1, Apoa2, Apoc1 and Apoc3, were up-regulated in the PFJ group. Several cytochrome P450 genes involved in phase I detoxification, such as Cyp1a2, Cyp2c67, Cyp2e1 and Cyp4f14, were also up-regulated. Three phase II detoxification genes, i.e. Ugt2b36, Cat and Gsto2, were up-regulated as well. On the other hand, genes down-regulated in the PFJ group include those involved in the insulin-signalling pathway, such as phosphatidylinositol kinases, Pik3r3 and Pi4ka, as well as mitogen-activated protein triple kinases, Map3k2 and Map3k11. Two genes related to fibrosis induction, Pcolce and Plod2, were also down-regulated in the PFJ group.

Functional enrichment analysis showed that various biological pathways (Table 5) and gene ontologies (biological processes) (Table 6) were differentially regulated in NRs given PFJ compared to controls. Among WikiPathways up-regulated by PFJ were those of tryptophan metabolism, methylation, fatty acid omega oxidation, nuclear receptors in lipid metabolism and toxicity, complement and coagulation cascades, urea cycle and metabolism of amino groups, retinol metabolism, metapathway biotransformation, one-carbon metabolism and nuclear receptors, as well as cytochrome P450s. Downregulated WikiPathways include regulation of actin cytoskeleton, insulin signalling and TNF-alpha NF- $\mathrm{\beta} \beta$ signalling. In relation to T2DM, a significant observation was that the insulin-signalling pathway was down-regulated in the PFJ group (Fig. 1).

Up-regulated gene ontologies (biological processes) of interest include negative feedback of very-low-density lipoprotein particle remodelling, negative feedback of receptor-mediated endocytosis, negative feedback of very-low-density lipoprotein particle clearance, negative feedback of lipid catabolic process, macromolecular 
Table 3 Diabetes assessment parameters of 3-week-old male NRs fed either a high-carbohydrate diet only or a highcarbohydrate diet supplemented with PFJ for 4 weeks

\begin{tabular}{|c|c|c|c|c|}
\hline \multirow[t]{3}{*}{ Group } & \multirow{2}{*}{\multicolumn{2}{|c|}{$\frac{\text { Control }}{(n=8)}$}} & \multirow{2}{*}{\multicolumn{2}{|c|}{$\frac{\text { PFJ }}{(n=8)}$}} \\
\hline & & & & \\
\hline & Mean & $\mathrm{SD}$ & Mean & SD \\
\hline \multicolumn{5}{|l|}{ Body weight (g) } \\
\hline Initial (3 weeks old) & 37 & 7 & 35 & 8 \\
\hline After 4 weeks & $77^{\mathrm{a}}$ & 8 & $70^{\mathrm{a}}$ & 10 \\
\hline \multicolumn{5}{|l|}{ Food intake } \\
\hline$g / d$ & $8^{\mathrm{a}}$ & 1 & $7^{\mathrm{a}}$ & 1 \\
\hline $\mathrm{kJ} / \mathrm{d}$ & $134^{\mathrm{a}}$ & 25 & $117^{a}$ & 13 \\
\hline $\mathrm{kcal} / \mathrm{d}$ & $32^{\mathrm{a}}$ & 6 & $28^{\mathrm{a}}$ & 3 \\
\hline $\begin{array}{l}\text { Food efficiency (g body weight gained/ } \\
1000 \mathrm{~kJ} \text { ) }\end{array}$ & 10.7 & 1.3 & 11.1 & 0.9 \\
\hline Fluid intake (ml/d) & 18 & 7 & 21 & 7 \\
\hline \multicolumn{5}{|l|}{ Random blood glucose (RBG) (mg/dl) } \\
\hline After four weeks & $241^{\mathrm{a}}$ & 133 & $128^{\mathrm{a}}$ & 121 \\
\hline \multicolumn{5}{|l|}{ Fasting blood glucose (FBG) (mg/dl) } \\
\hline After four weeks & 77 & 38 & 70 & 22 \\
\hline \multicolumn{5}{|l|}{ Terminal organ weight (\% body weight) } \\
\hline Liver & 3.6 & 0.6 & 3.6 & 0.5 \\
\hline Kidneys & 0.8 & 0.2 & 0.9 & 0.2 \\
\hline Caecum & $1.4^{\mathrm{a}}$ & 0.4 & $1.9^{\mathrm{a}}$ & 0.6 \\
\hline \multicolumn{5}{|l|}{ Adipose } \\
\hline Epididymal & $2.9^{a}$ & 0.5 & $2.4^{\mathrm{a}}$ & 0.8 \\
\hline Perirenal & $1.4^{\mathrm{a}}$ & 0.4 & $1.1^{\mathrm{a}}$ & 0.4 \\
\hline Brown fat & $1.7^{\mathrm{a}}$ & 0.2 & $1.5^{\mathrm{a}}$ & 0.3 \\
\hline Total fat & $6.0^{\mathrm{a}}$ & 0.8 & $5.1^{\mathrm{a}}$ & 1.1 \\
\hline Carcass & 73 & 2 & 75 & 5 \\
\hline Body length (cm) & $12.9^{\mathrm{a}}$ & 0.4 & $12.4^{\mathrm{a}}$ & 0.7 \\
\hline \multicolumn{5}{|l|}{ Plasma lipids (mmol/l) } \\
\hline Total cholesterol (TC) & 3.9 & 1.3 & 4.7 & 2.8 \\
\hline Triacylglycerol (TG) & $2.8^{\mathrm{a}}$ & 1.3 & $1.9^{\mathrm{a}}$ & 0.5 \\
\hline Insulin (pmol/l) & 0.6 & 0.3 & 0.6 & 0.4 \\
\hline
\end{tabular}

Values sharing a common superscript are significantly different from each other $(p<0.05)$ by two-tailed unpaired Student's $t$ test

complex remodelling, positive feedback of cholesterol esterification, negative feedback of lipid biosynthetic process, cellular response to lipid, cellular response to steroid hormone stimulus, negative feedback of cellular catabolic process, oxidation-reduction process, cellular response to peptide hormone stimulus and cellular carbohydrate metabolic process, as well as positive feedback of signal transduction. On the other hand, downregulated gene ontologies (biological processes) of interest include mammalian target of rapamycin (mTOR) signalling cascade, microtubule polymerisation, cell-cell junction organisation, cell activation involved in immune response, methylation, cell adhesion and catalytic activity, as well as negative feedback of protein phosphorylation.

Network analysis using the Ingenuity Pathways Analysis software showed that several apolipoproteins including apolipoproteins A1, A2 and C3 were upregulated by PFJ (Fig. 2). In addition, apolipoprotein $\mathrm{C} 1$ was up-regulated as well (Table 4).

\section{Real-time qRT-PCR validation}

To confirm the microarray results, the expression levels of six selected target genes were measured using realtime quantitative reverse transcription-polymerase chain reaction (qRT-PCR). From the four selected candidate reference genes tested, analysis using the geNorm 3.5 software [123] showed that Hpd, Nipbl and Trim39 were more stable than Cct6a. Hence, the former three were selected as the reference genes to normalise the expression values of the target genes. The directions of fold changes of the target genes obtained from the real-time qRT-PCR technique as quantified by the qBase software [48] were comparable to those obtained from the microarray technique (Fig. 3). However, the magnitudes of fold changes obtained using real-time qRT-PCR were consistently lower than those obtained using microarrays.

\section{Discussion}

Rapid economic progress has resulted in lifestyle changes, especially in diet and physical activity. In combination with aging populations, this has resulted in a worldwide epidemic of obesity, T2DM and metabolic syndrome [105]. In the USA, the prevalence of obesity which leads to T2DM and the metabolic syndrome has risen, even as the intake of fat is reduced. This has been referred to as the American Paradox [17], and highcarbohydrate intake has been suggested to be the cause of the problem [9].

Many phenolic-rich extracts have been suggested to be beneficial in preventing or treating T2DM and its related complications. In line with this, we have previously shown that providing PFJ at $1800 \mathrm{mg} / \mathrm{L}$ GAE ad libitum as the sole drinking fluid for 17 weeks blocked T2DM and metabolic syndrome progression in 12-week-old male NRs, as evidenced by normalisation of initially elevated blood glucose and plasma lipids $[15,16,100]$. The anti-diabetic effects of PFJ appeared relatively independent of starting age, and no impairment of energy intake or body weight dynamics have been observed in mature NRs, nor were any other toxic effects attributed to it [16]. In addition, PFJ protection against blood glucose elevation has also previously been shown to occur independently of diet (chow or semi-purified, moderate or high carbohydrate), study duration, initial blood glucose or application method [16]. PFJ may thus represent a 
Table $\mathbf{4}$ List of genes significantly regulated by PFJ

\begin{tabular}{|c|c|c|c|}
\hline Symbol & Definition & Differential score & Fold change \\
\hline \multicolumn{4}{|c|}{ Up-regulated genes } \\
\hline Sds & Serine dehydratase & 51.92 & 4.95 \\
\hline Plekhb1 & $\begin{array}{l}\text { Pleckstrin homology domain containing, } \\
\text { family B (evectins) member } 1\end{array}$ & 45.01 & 3.66 \\
\hline Npc1/1 & Niemann-Pick C1-like 1 & 30.36 & 7.41 \\
\hline$E G 240549$ & Predicted gene, EG240549 & 25.98 & 3.13 \\
\hline F7 & Coagulation factor VII & 23.44 & 3.08 \\
\hline Ecml & Extracellular matrix protein 1 & 23.18 & 2.32 \\
\hline Enpp2 & Ectonucleotide pyrophosphatase/phosphodiesterase 2 & 21.76 & 2.50 \\
\hline Ugt2b36 & UDP glucuronosyltransferase 2 family, polypeptide B36 & 21.09 & 2.98 \\
\hline Hdac3 & Histone deacetylase 3 & 20.37 & 2.93 \\
\hline Cspg5 & Chondroitin sulphate proteoglycan 5 & 20.27 & 2.05 \\
\hline Cyp2c67 & Cytochrome P450, family 2, subfamily c, polypeptide 67 & 20.06 & 14.08 \\
\hline Specc11 & SPECC1-like & 20.05 & 1.83 \\
\hline Cps 1 & $\begin{array}{l}\text { Carbamoyl-phosphate synthetase 1, nuclear } \\
\text { gene encoding mitochondrial protein XM_993466 }\end{array}$ & 19.42 & 2.78 \\
\hline$H b b-b 1$ & Haemoglobin, beta adult major chain & 19.19 & 2.14 \\
\hline Tnrc6a & Trinucleotide repeat containing $6 a$ & 19.03 & 1.58 \\
\hline Rps7 & Ribosomal protein S7 & 18.21 & 1.76 \\
\hline Apoc1 & Apolipoprotein C-I & 17.47 & 13.49 \\
\hline Cyp2e1 & Cytochrome P450, family 2, subfamily e, polypeptide 1 & 16.93 & 2.33 \\
\hline Ifrd1 & Interferon-related developmental regulator 1 & 16.84 & 1.94 \\
\hline Mup2 & Major urinary protein 2, transcript variant 1 & 16.51 & 197.62 \\
\hline Rpn2 & Ribophorin ॥ & 16.41 & 2.07 \\
\hline Asl & Argininosuccinate lyase & 16.33 & 1.85 \\
\hline Ptprt & Protein tyrosine phosphatase, receptor type, $T$ & 16.06 & 2.78 \\
\hline Bcdo2 & Beta-carotene 9', 10'-dioxygenase 2 & 16.00 & 2.43 \\
\hline Zfh $\times 2$ & Zinc finger homeobox 2 & 15.95 & 1.77 \\
\hline Mthfd1 & $\begin{array}{l}\text { Methylenetetrahydrofolate dehydrogenase } \\
\text { (NADP+ dependent), } \\
\text { methenyltetrahydrofolate cyclohydrolase, } \\
\text { formyltetrahydrofolate synthase }\end{array}$ & 15.95 & 1.54 \\
\hline Rnf215 & Ring finger protein 215 & 15.91 & 1.63 \\
\hline Gne & Glucosamine & 15.82 & 2.54 \\
\hline Cyp4f14 & Cytochrome P450, family 4, subfamily f, polypeptide 14 & 15.60 & 2.55 \\
\hline$Z x d a$ & Zinc finger, $\mathrm{X}$-linked, duplicated A & 15.35 & 1.51 \\
\hline Nat1 & $\mathrm{N}$-acetyltransferase 1 (arylamine $\mathrm{N}$-acetyltransferase) & 15.26 & 2.05 \\
\hline Cat & Catalase & 15.23 & 2.84 \\
\hline Tyms-ps & Thymidylate synthase, pseudogene & 15.16 & 1.69 \\
\hline F5 & Coagulation factor $V$ & 15.12 & 2.38 \\
\hline Fbxo7 & F-box only protein 7 & 14.96 & 1.71 \\
\hline Apoa2 & Apolipoprotein A-II & 14.91 & 2.67 \\
\hline Hagh & Hydroxyacyl glutathione hydrolase & 14.87 & 1.64 \\
\hline Alas 1 & Aminolevulinic acid synthase 1 & 14.80 & 10.33 \\
\hline Inmt & Indolethylamine $\mathrm{N}$-methyltransferase & 14.79 & 2.62 \\
\hline 620807.00 & Predicted gene, 620807 & 14.78 & 106.29 \\
\hline
\end{tabular}


Table 4 List of genes significantly regulated by PFJ (Continued)

\begin{tabular}{|c|c|c|c|}
\hline \\
\hline Hsd17b10 & $\begin{array}{l}\text { Hydroxysteroid (17-beta) dehydrogenase } \\
\text { 10, nuclear gene encoding mitochondrial protein }\end{array}$ & 14.75 & 2.30 \\
\hline Nrii3 & Nuclear receptor subfamily 1, group I, member 3 & 14.63 & 2.02 \\
\hline Nit2 & Nitrilase family, member 2 & 14.58 & 1.99 \\
\hline Tbcld15 & TBC1 domain family, member 15 & 14.57 & 1.71 \\
\hline Apoc3 & Apolipoprotein C-III & 14.56 & 2.77 \\
\hline ORF61 & Open reading frame 61 & 14.51 & 1.54 \\
\hline Ephx1 & Epoxide hydrolase 1, microsomal & 14.36 & 2.98 \\
\hline Serpinald & $\begin{array}{l}\text { Serine (or cysteine) peptidase inhibitor, } \\
\text { clade A, member } 1 d\end{array}$ & 14.26 & 13.47 \\
\hline Stab1 & Stabilin 1 & 14.19 & 2.00 \\
\hline Ifitm2 & Interferon induced transmembrane protein 2 & 14.05 & 1.55 \\
\hline Hmgcs2 & $\begin{array}{l}\text { 3-hydroxy-3-methylglutaryl-Coenzyme A } \\
\text { synthase 2, nuclear gene encoding mitochondrial protein }\end{array}$ & 14.03 & 5.41 \\
\hline Serpina1b & Serine (or cysteine) preptidase inhibitor, clade A, member $1 \mathrm{~b}$ & 14.03 & 13.48 \\
\hline Tmem132e & Transmembrane protein $132 \mathrm{E}$ & 13.98 & 1.99 \\
\hline Syvn1 & Synovial apoptosis inhibitor 1, synoviolin & 13.97 & 1.78 \\
\hline Cypla2 & Cytochrome P450, family 1, subfamily a, polypeptide 2 & 13.92 & 2.38 \\
\hline Reln & Reelin & 13.90 & 2.44 \\
\hline$F z d 7$ & Frizzled homologue 7 (Drosophila) & 13.87 & 1.96 \\
\hline$F 13 b$ & Coagulation factor XIII, beta subunit & 13.83 & 2.30 \\
\hline Rpl36al & Ribosomal protein I36a-like & 13.73 & 1.79 \\
\hline Klkbl & Kallikrein B, plasma 1 & 13.72 & 2.29 \\
\hline Sdf2 & Stromal cell derived factor 2 & 13.53 & 1.44 \\
\hline $3110049 J 23 R i k$ & RIKEN cDNA 3110049 J23 gene & 13.44 & 1.70 \\
\hline 2810004N20Rik & RIKEN cDNA 2810004 N20 gene & 13.40 & 1.74 \\
\hline Rxrg & Retinoid $X$ receptor gamma & 13.36 & 2.29 \\
\hline Ces3 & Carboxylesterase 3 & 13.20 & 4.77 \\
\hline $\sec 16 b$ & SEC16 homologue B (Saccharomyces cerevisiae) & 13.20 & 2.31 \\
\hline Gsto2 & Glutathione S-transferase omega 2 & 13.17 & 2.33 \\
\hline 5830404H04Rik & RIKEN cDNA 5830404H04 gene & 13.14 & 1.90 \\
\hline Creld 1 & Cysteine-rich with EGF-like domains 1 & 13.13 & 1.46 \\
\hline Matia & Methionine adenosyltransferase I, alpha & 13.04 & 14.39 \\
\hline Apoa1 & Apolipoprotein A-I & 13.02 & 25.82 \\
\hline \multicolumn{4}{|c|}{ Down-regulated genes } \\
\hline St3gal6 & ST3 beta-galactoside alpha-2,3-sialyltransferase 6 & -13.09 & -21.14 \\
\hline Btbd3 & BTB (POZ) domain containing 3 & -13.12 & -2.51 \\
\hline Wbp2 & WW domain binding protein 2 & -13.12 & -1.47 \\
\hline LOC100045542 & $\begin{array}{l}\text { Predicted: similar to FERMRhoGEF (Arhgef) } \\
\text { and pleckstrin domain protein } 1\end{array}$ & -13.14 & -3.04 \\
\hline Shmt2 & $\begin{array}{l}\text { Serine hydroxymethyltransferase } 2 \text { (mitochondrial), } \\
\text { nuclear gene encoding mitochondrial protein }\end{array}$ & -13.19 & -1.69 \\
\hline Clptmil & CLPTM1-like & -13.24 & -1.48 \\
\hline $\operatorname{Cox} 10$ & $\begin{array}{l}\text { COX10 homologue, cytochrome c oxidase assembly } \\
\text { protein, heme A: farnesyltransferase (yeast), nuclear } \\
\text { gene encoding mitochondrial protein }\end{array}$ & -13.26 & -1.54 \\
\hline Gpr107 & G protein-coupled receptor 107 & -13.27 & -1.82 \\
\hline
\end{tabular}


Table 4 List of genes significantly regulated by PFJ (Continued)

\begin{tabular}{|c|c|c|c|}
\hline Dnajc10 & Dnaj (Hsp40) homologue, subfamily C, member 10 & -13.29 & -1.55 \\
\hline Plod2 & Procollagen-lysine, 2-oxoglutarate 5-dioxygenase 2 & -13.29 & -2.74 \\
\hline Magee1 & Melanoma antigen, family $E_{1} 1$ & -13.31 & -4.06 \\
\hline Ppp1r16a & Protein phosphatase 1, regulatory (inhibitor) subunit 16A & -13.35 & -2.19 \\
\hline Prkcbp 1 & Protein kinase $\mathrm{C}$ binding protein 1 & -13.36 & -2.04 \\
\hline Map3k11 & Mitogen-activated protein kinase kinase kinase 11 & -13.37 & -1.48 \\
\hline Marcks & Myristoylated alanine rich protein kinase $C$ substrate & -13.38 & -1.47 \\
\hline Tex9 & Testis expressed gene 9 & -13.39 & -2.46 \\
\hline $\operatorname{Cog} 1$ & Component of oligomeric golgi complex 1 & -13.40 & -1.51 \\
\hline Slc39a13 & Solute carrier family 39 (metal ion transporter), member 13 & -13.40 & -2.66 \\
\hline Fam110b & Family with sequence similarity 110 , member $B$ & -13.43 & -3.23 \\
\hline Cox6b1 & Cytochrome c oxidase, subunit Vlb polypeptide 1 & -13.44 & -1.45 \\
\hline Stxbp2 & Syntaxin binding protein 2 & -13.45 & -1.68 \\
\hline Ino80b & INO80 complex subunit B & -13.45 & -2.52 \\
\hline Nap $1 / 4$ & Nucleosome assembly protein 1-like 4 & -13.45 & -1.55 \\
\hline Flii & Flightless I homologue (Drosophila) & -13.47 & -1.63 \\
\hline Ahdcl & AT hook, DNA binding motif, containing 1 & -13.55 & -1.63 \\
\hline Nol5a & Nucleolar protein $5 \mathrm{~A}$ & -13.69 & -1.52 \\
\hline 2400001E08Rik & RIKEN cDNA 2400001 E08 gene & -13.75 & -1.77 \\
\hline Prmt5 & Protein arginine $\mathrm{N}$-methyltransferase 5 & -13.77 & -1.79 \\
\hline Tinagl & Tubulointerstitial nephritis antigen-like & -13.78 & -3.14 \\
\hline Parl & Presenilin associated, rhomboid-like & -13.84 & -1.51 \\
\hline Zmat5 & Zinc finger, matrin type 5 & -13.85 & -1.86 \\
\hline Calm3 & Calmodulin 3 & -13.86 & -2.15 \\
\hline$A k 3 / 1$ & $\begin{array}{l}\text { Adenylate kinase } 3 \text {-like 1, nuclear gene } \\
\text { encoding mitochondrial protein }\end{array}$ & -13.86 & -1.49 \\
\hline 2700087H15Rik & RIKEN cDNA 2700087H15 gene & -13.93 & -1.54 \\
\hline Grit & RHOGTPase-activating protein & -13.95 & -2.23 \\
\hline X99384 & cDNA sequence X99384 & -13.96 & -1.77 \\
\hline$D d \times 27$ & DEAD (Asp-Glu-Ala-Asp) box polypeptide 27 & -13.97 & -2.09 \\
\hline Zfp313 & Zinc finger protein 313 & -13.98 & -1.53 \\
\hline D15Wsu169e & $\begin{array}{l}\text { DNA segment, Chr } 15 \text {, Wayne State } \\
\text { University } 169 \text {, expressed }\end{array}$ & -14.02 & -4.17 \\
\hline Zer1 & Zer-1 homologue (Caenorhabditis elegans) & -14.03 & -2.01 \\
\hline Snapc2 & Small nuclear RNA activating complex, polypeptide 2 & -14.05 & -2.04 \\
\hline Dock1 & Dedicator of cytokinesis 1 & -14.19 & -1.91 \\
\hline Pak4 & P21 (CDKN1A)-activated kinase 4 & -14.21 & -1.51 \\
\hline Arl2 & ADP-ribosylation factor-like 2 & -14.22 & -6.24 \\
\hline Pcolce & Procollagen C-endopeptidase enhancer protein & -14.24 & -2.15 \\
\hline 1110018 G07Rik & RIKEN cDNA $1110018 \mathrm{G} 07$ gene & -14.28 & -1.61 \\
\hline 2610528J11Rik & RIKEN cDNA 2610528J11 gene & -14.29 & -2.35 \\
\hline Akp2 & Alkaline phosphatase 2, liver & -14.31 & -2.72 \\
\hline Mapre1 & Microtubule-associated protein, RP/EB family, member 1 & -14.35 & -1.56 \\
\hline Tmem138 & Transmembrane protein 138 & -14.36 & -2.51 \\
\hline Pacs2 & Phosphofurin acidic cluster sorting protein 2 & -14.41 & -1.70 \\
\hline LOC100047173 & PREDICTED: similar to synaptotagmin-like 1 & -14.41 & -3.34 \\
\hline
\end{tabular}


Table 4 List of genes significantly regulated by PFJ (Continued)

\begin{tabular}{|c|c|c|c|}
\hline Ano10 & Anoctamin 10 & -14.47 & -5.94 \\
\hline Vasn & Vasorin & -14.48 & -1.65 \\
\hline $\mathrm{Cm} / 4$ & Camello-like 4 & -14.50 & -3.02 \\
\hline Clcn3 & Chloride channel 3, transcript variant a & -14.50 & -1.73 \\
\hline Pik3r3 & $\begin{array}{l}\text { Phosphatidylinositol 3-kinase, regulatory } \\
\text { subunit, polypeptide } 3 \text { (p55) }\end{array}$ & -14.54 & -4.60 \\
\hline Timp 1 & TIMP metallopeptidase inhibitor 1 & -14.61 & -1.60 \\
\hline Fbx/15 & F-box and leucine-rich repeat protein 15 & -14.65 & -1.59 \\
\hline$N p c 2$ & Niemann-Pick disease, type C2 & -14.68 & -1.60 \\
\hline Mrps33 & $\begin{array}{l}\text { Mitochondrial ribosomal protein } \mathrm{S3} \text {, nuclear } \\
\text { gene encoding mitochondrial protein, } \\
\text { transcript variant } 2\end{array}$ & -14.73 & -1.65 \\
\hline Pgam5 & Phosphoglycerate mutase family member 5 & -14.73 & -1.84 \\
\hline 2310005N01Rik & RIKEN cDNA 2310005N01 gene & -14.79 & -2.67 \\
\hline Ctdspl & $\begin{array}{l}\text { CTD (carboxy-terminal domain, RNA polymerase II, } \\
\text { polypeptide A) small phosphatase-like }\end{array}$ & -14.83 & -2.49 \\
\hline LOC100046039 & PREDICTED: similar to histone deacetylase HD1 & -14.85 & -2.29 \\
\hline Gnptab & $\begin{array}{l}\mathrm{N} \text {-acetylglucosamine-1-phosphate transferase, } \\
\text { alpha and beta subunits }\end{array}$ & -14.93 & -1.90 \\
\hline Tbcld14 & TBC1 domain family, member 14 & -15.03 & -2.88 \\
\hline Cyr61 & Cysteine-rich protein 61 & -15.07 & -4.37 \\
\hline Gdpd1 & $\begin{array}{l}\text { Glycerophosphodiester phosphodiesterase } \\
\text { domain containing } 1\end{array}$ & -15.11 & -1.58 \\
\hline 2310022B05Rik & RIKEN cDNA 2310022B05 gene & -15.16 & -1.53 \\
\hline Asnal & $\begin{array}{l}\text { Arsa arsenite transporter, ATP-binding, } \\
\text { homologue } 1 \text { (bacterial) }\end{array}$ & -15.16 & -1.66 \\
\hline Tcf4 & Transcription factor 4, transcript variant 1 & -15.17 & -2.10 \\
\hline Vps26b & Vacuolar protein sorting 26 homologue B (yeast) & -15.43 & -1.57 \\
\hline Nf2 & Neurofibromatosis 2 & -15.54 & -2.64 \\
\hline LOC192758 & Similar to hypothetical protein MGC39650 & -15.63 & -3.10 \\
\hline Drg2 & Developmentally regulated GTP binding protein 2 & -15.66 & -1.74 \\
\hline lagap 1 & IQ motif containing GTPase activating protein 1 & -15.89 & -1.73 \\
\hline Nrp1 & Neuropilin 1 & -16.05 & -2.33 \\
\hline Tbcld13 & TBC1 domain family, member 13 & -16.13 & -3.24 \\
\hline 2310003P10Rik & RIKEN cDNA 2310003P10 gene & -16.15 & -3.82 \\
\hline Trim28 & Tripartite motif protein 28 & -16.18 & -1.79 \\
\hline T/r2 & Toll-like receptor 2 & -16.41 & -2.26 \\
\hline 0910001L09Rik & RIKEN cDNA 0910001L09 gene & -16.42 & -2.15 \\
\hline B930041F14Rik & RIKEN cDNA B930041F14 gene & -16.74 & -2.44 \\
\hline Nup93 & Nucleoporin 93 kDa & -16.93 & -2.22 \\
\hline Lphn1 & Latrophilin 1 & -17.11 & -2.08 \\
\hline Odz4 & Odd Oz/ten-m homologue 4 (Drosophila) & -17.13 & -4.10 \\
\hline Gnai2 & Guanine nucleotide binding protein, alpha inhibiting 2 & -17.14 & -2.08 \\
\hline Cyp4f13 & Cytochrome P450, family 4, subfamily f, polypeptide 13 & -17.16 & -4.76 \\
\hline Aacs & Acetoacetyl-coa synthetase & -17.26 & -1.62 \\
\hline Smarca4 & $\begin{array}{l}\text { SWI/SNF related, matrix associated, actin } \\
\text { dependent regulator of chromatin, } \\
\text { subfamily a, member } 4\end{array}$ & -17.42 & -1.89 \\
\hline
\end{tabular}


Table 4 List of genes significantly regulated by PFJ (Continued)

\begin{tabular}{llll}
\hline Gatad2b & GATA zinc finger domain containing 2B & -17.46 & -2.31 \\
Actr1b & ARP1 actin-related protein 1 homologue & -17.87 & -1.74 \\
& B, centractin beta (yeast) & -17.93 & -18.31 \\
Neo1 & Neogenin 1 & -18.00 \\
Meis2 & Meis homeobox 2, transcript variant 2 & -18.44 & -1.91 \\
Serpinh1 & Serine (or cysteine) peptidase inhibitor, clade H, member 1 & -18.44 & -9.72 \\
Cc2d2a & Coiled-coil and C2 domain containing 2A & -18.88 & -2.28 \\
Vdac1 & Voltage-dependent anion channel 1 & -19.13 & -1.65 \\
Picalm & Phosphatidylinositol binding clathrin assembly protein & -19.20 & -1.73 \\
Ankrd24 & Ankyrin repeat domain 24 & -19.52 & -6.41 \\
Pi4ka & Phosphatidylinositol 4-kinase, catalytic, alpha polypeptide & -19.53 \\
Map3k2 & Mitogen-activated protein kinase kinase kinase 2 & -19.78 & -2.19 \\
1700029G01Rik & RIKEN cDNA 1700029G01 gene & -21.42 & -3.51 \\
Atn1 & Atrophin 1 & -22.26 \\
Itprip & Inositol 1,4,5-triphosphate receptor interacting protein & -2.21 \\
Gadd45g & Growth arrest and DNA-damage-inducible 45 gamma & -23.65 & -1.86 \\
Ly6e & Lymphocyte antigen 6 complex, locus E & -23.91 \\
Ctcfl & CCCTC-binding factor (zinc finger protein)-like & -27.64 \\
\hline
\end{tabular}

source for food supplementation or as a nutraceutical having possible anti-diabetic properties.

\section{PFJ reduced weight gain, adipose tissue, plasma TG and} plasma RBG but increased caecum weight

Following the 4-week high-carbohydrate diet challenge in weanling male NRs, the group supplemented with PFJ weighed less and their food intake was significantly lower. However, the carcass (lean mass) and food efficiency tended to be greater for the PFJ group, and they had less adipose tissue. Thus, control rats gained more weight than those in the PFJ group, mostly due to the accumulation of adipose tissue, while PFJ seemed to inhibit appetite and reduce body fat percentage without reducing food efficiency or leading to a decrease in lean body mass. The latter effect is a characteristic of dietary fibres that are fermented by large bowel microbiota [107], and it is noteworthy that the enlarged caeca in rats fed PFJ would be consistent with enhanced fermentation of PFJ components by their large bowel flora. Faster weight gain in male NRs has also been found to enhance T2DM induction in growing rats $[15,21]$. As visceral adiposity and hyperlipaemia are two of the risk factors for cardiovascular insults in metabolic syndrome, the reduced body fat percentage and TG levels observed in the PFJ group indicate a beneficial metabolic effect beyond improved blood glucose levels.

The PFJ group also had a significantly lower level of RBG compared to the controls, although no differences were observed in terms of FBG. RBG is an early and more reliable parameter of T2DM than FBG in NRs
[14-16, 21, 85]. This is because the correlations between circulating glucose and different markers of T2DM, such as elevations in $\mathrm{HbA} 1 \mathrm{c}$ and hypertension, are stronger for RBG than FBG in NRs. In addition, acute cell and organ damage is best reflected by the degree and duration of postprandial hyperglycaemia, thus rendering RBG the best indicator of such damage $[15,16]$. The observation that insulin levels were not significantly different between the two groups $(p>0.05)$ indicates that the improved glucose control was due to mechanisms other than increased insulin secretion, such as reduced intestinal glucose absorption or improved insulin sensitivity. As hyperinsulinaemia is one of the first indicators of insulin resistance and a risk factor for the eventual depletion of pancreatic beta cells, this is a crucial observation for the prevention of T2DM, potentially reducing the need for or delaying the onset of insulin therapy or enabling a reduced dose. PFJ thus exerted beneficial metabolic effects, preventing NRs from overconsumption of calories and achieving improved control of plasma glucose and lipids.

As NRs in the present study were fed ad libitum, at least part of the effects ascribed to PFJ could be due to mild caloric restriction caused by reduced food intake. Nevertheless, caloric restriction in the classical sense typically entails a $20-40 \%$ reduction in food consumption relative to normal intake [64], which was not the case here at 15-20\%. Furthermore, we previously found no reduction in food intake or any difference in PFJ protection in older NRs when given artificially sweetened PFJ, suggesting that PFJ protection against diabetes 
Table $\mathbf{5}$ List of WikiPathways significantly regulated by PFJ

\begin{tabular}{|c|c|c|c|c|}
\hline WikiPathway name & No. changed & $\%$ changed & $Z$ score & Permuted $p$ \\
\hline \multicolumn{5}{|l|}{ Up-regulated WikiPathways } \\
\hline Tryptophan metabolism:WP79 & 6 & 15.3846 & 9.4735 & 0.0000 \\
\hline Aflatoxin B1 metabolism:WP1262 & 2 & 40.0000 & 9.1192 & 0.0005 \\
\hline Methylation:WP1247 & 2 & 28.5714 & 7.6363 & 0.0000 \\
\hline Fatty acid omega oxidation:WP33 & 2 & 28.5714 & 7.6363 & 0.0015 \\
\hline Statin pathway (PharmGKB):WP1 & 3 & 16.6667 & 6.9838 & 0.0000 \\
\hline Blood clotting cascade:WP460 & 3 & 15.7895 & 6.7763 & 0.0000 \\
\hline Nuclear receptors in lipid metabolism and toxicity:WP431 & 3 & 10.0000 & 5.2068 & 0.0005 \\
\hline Complement and coagulation cascades:WP449 & 4 & 6.8966 & 4.7841 & 0.0000 \\
\hline Urea cycle and metabolism of amino groups:WP426 & 2 & 11.1111 & 4.5187 & 0.0020 \\
\hline Retinol metabolism:WP1259 & 3 & 7.6923 & 4.4328 & 0.0005 \\
\hline Metapathway biotransformation:WP1251 & 5 & 4.4248 & 3.9468 & 0.0000 \\
\hline One-carbon metabolism:WP435 & 2 & 8.3333 & 3.7980 & 0.0040 \\
\hline Nuclear receptors:WP509 & 2 & 5.5556 & 2.9123 & 0.0075 \\
\hline Cytochrome P450:WP1274 & 2 & 5.2632 & 2.8039 & 0.0105 \\
\hline \multicolumn{5}{|l|}{ Down-Regulated WikiPathways } \\
\hline Regulation of actin cytoskeleton:WP523 & 4 & 3.0534 & 3.6287 & 0.0080 \\
\hline Insulin signalling:WP65 & 4 & 2.8169 & 3.4170 & 0.0110 \\
\hline Endochondral ossification:WP1270 & 2 & 3.3898 & 2.7401 & 0.0460 \\
\hline TNF-alpha NF-Kß signalling pathway:WP246 & 3 & 2.2222 & 2.4278 & 0.0430 \\
\hline
\end{tabular}

development does not depend on reductions in food consumption [16].

In addition, NRs in the PFJ group had heavier caeca $(p<0.05)$ than the controls. This may be attributed to the presence of fermentable dietary fibres in the PFJ extract that resisted upper gut digestion and reached the caecum (the main site of bacterial fermentation in rodents) where they were fermented by the microbiota. However, the bioactive components in PFJ and/or their derived metabolites may have also played a part in the observed caecum enlargement. In the colon, where microbial glucosidases and glucuronidases are active, phenolic glycosides are intensively metabolised and their metabolites also modify colon parameters, such as shortchain fatty acids, amino acids and vitamins [30]. This is in agreement with the results of others, where increased caecal weight was observed in rats fed diets containing polyphenols [2, 37, 53]. Romo-Vaquero et al. [95] also reported that rosemary extract enriched in the bioactive compound carnosic acid caused caecum enlargement in female Zucker rats. The presence of non-digested materials fermented by large bowel microbiota might have caused the enlarged caeca. The same study also reported that the rosemary extract lowered body weights, serum lipids and insulin levels in the rats and partially attributed this to the inhibition of a pre-duodenal butyrate esterase activity [95]. Thus, the lower adipose tissue content and body weights of the NRs on PFJ may also have been a consequence of the inhibition of specific enzymes in the gut. A pomegranate extract, rich in punicalagin and ellagic acid, also increased caecum size and Bifidobacterium in mice [84]. The gut microbiota can modulate host energy metabolism and is thus a significant contributor to the development of obesity and metabolic disorders [130].

\section{Microarray gene expression analysis revealed down-} regulation of the insulin-signalling pathway linked to altered insulin availability

Research on the health effects of plant-based foods will benefit from taking a holistic approach to understand the plethora of effects mediated by a range of bioactive metabolites derived from plant consumption. Thus, the combination of different 'omics' profiling techniques in the concept of systems biology, or nutrigenomics as termed in the context of nutrition-related sciences, would be important for this purpose [47]. In the present study, microarrays delineated hepatic gene expression differences between young NRs supplemented with PFJ or not and further confirmed several target genes of interest using real-time qRT-PCR.

In relation to $\mathrm{T} 2 \mathrm{DM}$, the most significant observation from the functional enrichment analysis of the microarray gene expression data was that the insulin- 
Table 6 List of gene ontologies (biological processes) significantly regulated by PFJ

\begin{tabular}{|c|c|c|c|c|c|}
\hline GO ID & GO name & No. changed & $\%$ changed & Z score & Permuted $p$ \\
\hline \multicolumn{6}{|c|}{ Up-regulated gene ontologies (biological processes) } \\
\hline GO:0010903 & $\begin{array}{l}\text { Negative regulation of very-low-density lipoprotein } \\
\text { particle remodelling }\end{array}$ & 3 & 100.0000 & 26.2353 & 0.0000 \\
\hline GO:0060192 & Negative regulation of lipase activity & 4 & 40.0000 & 19.0387 & 0.0000 \\
\hline GO:0033700 & Phospholipid efflux & 4 & 33.3333 & 17.3429 & 0.0000 \\
\hline GO:0060416 & Response to growth hormone stimulus & 4 & 30.7692 & 16.6448 & 0.0000 \\
\hline GO:0032488 & Cdc42 protein signal transduction & 2 & 50.0000 & 15.0814 & 0.0000 \\
\hline GO:0046461 & Neutral lipid catabolic process & 3 & 33.3333 & 15.0179 & 0.0000 \\
\hline GO:0042157 & Lipoprotein metabolic process & 5 & 20.0000 & 14.8938 & 0.0000 \\
\hline GO:0007494 & Midgut development & 3 & 30.0000 & 14.2268 & 0.0000 \\
\hline GO:0048261 & Negative regulation of receptor-mediated endocytosis & 2 & 40.0000 & 13.4602 & 0.0000 \\
\hline GO:0010915 & Regulation of very-low-density lipoprotein particle clearance & 2 & 40.0000 & 13.4602 & 0.0000 \\
\hline GO:0071825 & Protein-lipid complex subunit organisation & 4 & 19.0476 & 12.9842 & 0.0000 \\
\hline GO:0015918 & Sterol transport & 5 & 14.2857 & 12.4800 & 0.0000 \\
\hline GO:0050995 & Negative regulation of lipid catabolic process & 3 & 23.0769 & 12.4241 & 0.0005 \\
\hline GO:0030300 & Regulation of intestinal cholesterol absorption & 2 & 33.3333 & 12.2609 & 0.0000 \\
\hline GO:0034381 & Plasma lipoprotein particle clearance & 3 & 21.4286 & 11.9549 & 0.0000 \\
\hline GO:0008203 & Cholesterol metabolic process & 7 & 9.5890 & 11.9276 & 0.0000 \\
\hline GO:0034367 & Macromolecular complex remodelling & 3 & 20.0000 & 11.5328 & 0.0000 \\
\hline GO:0010873 & Positive regulation of cholesterol esterification & 2 & 28.5714 & 11.3268 & 0.0000 \\
\hline GO:0018904 & Organic ether metabolic process & 7 & 8.6420 & 11.2675 & 0.0000 \\
\hline GO:0071941 & Nitrogen cycle metabolic process & 2 & 22.2222 & 9.9459 & 0.0000 \\
\hline GO:0051055 & Negative regulation of lipid biosynthetic process & 3 & 13.6364 & 9.4265 & 0.0000 \\
\hline GO:0071320 & Cellular response to cyclic adenosine monophosphate & 2 & 20.0000 & 9.4149 & 0.0005 \\
\hline GO:0042632 & Cholesterol homeostasis & 4 & 10.0000 & 9.2153 & 0.0000 \\
\hline GO:0071396 & Cellular response to lipid & 3 & 13.0435 & 9.2059 & 0.0005 \\
\hline GO:0071383 & Cellular response to steroid hormone stimulus & 5 & 6.4935 & 8.1091 & 0.0000 \\
\hline GO:0006720 & Isoprenoid metabolic process & 4 & 7.0175 & 7.5752 & 0.0000 \\
\hline GO:0050817 & Coagulation & 4 & 5.8824 & 6.8498 & 0.0000 \\
\hline GO:0001101 & Response to acid & 4 & 5.7971 & 6.7922 & 0.0005 \\
\hline GO:0010243 & Response to organic nitrogen & 5 & 4.7170 & 6.7315 & 0.0000 \\
\hline GO:0044272 & Sulphur compound biosynthetic process & 3 & 7.3171 & 6.7133 & 0.0015 \\
\hline GO:0017144 & Drug metabolic process & 2 & 10.5263 & 6.6960 & 0.0045 \\
\hline GO:0033762 & Response to glucagon stimulus & 3 & 6.9767 & 6.5356 & 0.0005 \\
\hline GO:0044106 & Cellular amine metabolic process & 9 & 2.7692 & 6.4744 & 0.0000 \\
\hline GO:0043436 & Oxoacid metabolic process & 12 & 2.1053 & 6.1883 & 0.0000 \\
\hline GO:0033574 & Response to testosterone stimulus & 2 & 9.0909 & 6.1811 & 0.0030 \\
\hline GO:0009636 & Response to toxin & 4 & 4.7619 & 6.0507 & 0.0010 \\
\hline GO:0031100 & Organ regeneration & 3 & 5.8824 & 5.9287 & 0.0010 \\
\hline GO:0042743 & Hydrogen peroxide metabolic process & 2 & 8.3333 & 5.8913 & 0.0045 \\
\hline GO:0031667 & Response to nutrient levels & 7 & 2.7237 & 5.6322 & 0.0000 \\
\hline GO:0051262 & Protein tetramerisation & 3 & 5.3571 & 5.6146 & 0.0020 \\
\hline GO:0031330 & Negative regulation of cellular catabolic process & 2 & 7.1429 & 5.4051 & 0.0060 \\
\hline GO:0030193 & Regulation of blood coagulation & 2 & 6.8966 & 5.2990 & 0.0060 \\
\hline
\end{tabular}


Table 6 List of gene ontologies (biological processes) significantly regulated by PFJ (Continued)

\begin{tabular}{|c|c|c|c|c|c|}
\hline GO:0010043 & Response to zinc ion & 2 & 6.8966 & 5.2990 & 0.0050 \\
\hline GO:0031647 & Regulation of protein stability & 3 & 4.8387 & 5.2867 & 0.0020 \\
\hline GO:0051384 & Response to glucocorticoid stimulus & 4 & 3.3898 & 4.9035 & 0.0010 \\
\hline GO:0007623 & Circadian rhythm & 3 & 4.2254 & 4.8711 & 0.0040 \\
\hline GO:0055114 & Oxidation-reduction process & 11 & 1.6129 & 4.7935 & 0.0000 \\
\hline GO:0071375 & Cellular response to peptide hormone stimulus & 4 & 3.1008 & 4.6273 & 0.0035 \\
\hline GO:0006725 & Cellular aromatic compound metabolic process & 4 & 2.9851 & 4.5123 & 0.0015 \\
\hline GO:0033013 & Tetrapyrrole metabolic process & 2 & 5.1282 & 4.4651 & 0.0070 \\
\hline GO:0051186 & Cofactor metabolic process & 5 & 2.4510 & 4.4123 & 0.0005 \\
\hline GO:0033555 & Multicellular organismal response to stress & 2 & 4.3478 & 4.0441 & 0.0110 \\
\hline GO:0044262 & Cellular carbohydrate metabolic process & 6 & 1.8127 & 3.8583 & 0.0045 \\
\hline GO:0061061 & Muscle structure development & 2 & 3.8462 & 3.7493 & 0.0190 \\
\hline GO:0042445 & Hormone metabolic process & 3 & 2.7027 & 3.6494 & 0.0100 \\
\hline GO:0042493 & Response to drug & 5 & 1.8116 & 3.5138 & 0.0045 \\
\hline GO:0006730 & One-carbon metabolic process & 4 & 1.9802 & 3.3655 & 0.0125 \\
\hline GO:0007626 & Locomotory behaviour & 3 & 2.3810 & 3.3384 & 0.0125 \\
\hline GO:0014070 & Response to organic cyclic compound & 4 & 1.9417 & 3.3146 & 0.0065 \\
\hline GO:0048513 & Organ development & 9 & 1.1704 & 3.1890 & 0.0050 \\
\hline GO:0009607 & Response to biotic stimulus & 6 & 1.4458 & 3.1796 & 0.0090 \\
\hline GO:0009611 & Response to wounding & 5 & 1.6026 & 3.1729 & 0.0120 \\
\hline GO:0009791 & Post-embryonic development & 2 & 2.7778 & 3.0325 & 0.0375 \\
\hline GO:0006414 & Translational elongation & 2 & 2.6316 & 2.9216 & 0.0485 \\
\hline GO:0010466 & Negative regulation of peptidase activity & 3 & 1.9868 & 2.9171 & 0.0325 \\
\hline GO:0050679 & Positive regulation of epithelial cell proliferation & 2 & 2.4096 & 2.7455 & 0.0480 \\
\hline GO:0035335 & Peptidyl-tyrosine dephosphorylation & 2 & 2.3529 & 2.6988 & 0.0380 \\
\hline GO:0034284 & Response to monosaccharide stimulus & 2 & 2.3529 & 2.6988 & 0.0490 \\
\hline GO:0032989 & Cellular component morphogenesis & 4 & 1.4652 & 2.6156 & 0.0285 \\
\hline GO:0009967 & Positive regulation of signal transduction & 5 & 1.1876 & 2.3859 & 0.0305 \\
\hline \multicolumn{6}{|c|}{ Down-regulated gene ontologies (biological processes) } \\
\hline GO:0032006 & Regulation of mTORsignalling cascade & 2 & 12.5000 & 6.1613 & 0.0040 \\
\hline GO:0031113 & Regulation of microtubule polymerisation & 2 & 11.1111 & 5.7727 & 0.0070 \\
\hline GO:0001702 & Gastrulation with mouth forming second & 2 & 9.0909 & 5.1559 & 0.0075 \\
\hline GO:0045216 & Cell-cell junction organisation & 2 & 5.2632 & 3.7231 & 0.0245 \\
\hline GO:0042632 & Cholesterol homeostasis & 2 & 5.0000 & 3.6045 & 0.0235 \\
\hline GO:0006793 & Phosphorus metabolic process & 14 & 1.4433 & 3.5098 & 0.0015 \\
\hline GO:0031214 & Biomineral tissue development & 2 & 4.5455 & 3.3902 & 0.0280 \\
\hline GO:0002263 & Cell activation involved in immune response & 2 & 4.5455 & 3.3902 & 0.0320 \\
\hline GO:0042475 & Odontogenesis of dentine-containing tooth & 2 & 4.4444 & 3.3408 & 0.0315 \\
\hline GO:0032259 & Methylation & 4 & 2.5157 & 3.1405 & 0.0145 \\
\hline GO:0030155 & Regulation of cell adhesion & 4 & 2.4096 & 3.0318 & 0.0160 \\
\hline GO:0050790 & Regulation of catalytic activity & 15 & 1.2490 & 3.0295 & 0.0080 \\
\hline GO:0010243 & Response to organic nitrogen & 3 & 2.8302 & 2.9800 & 0.0270 \\
\hline GO:0001933 & Negative regulation of protein phosphorylation & 2 & 3.6364 & 2.9179 & 0.0460 \\
\hline GO:0071841 & Cellular component organisation or biogenesis at cellular level & 18 & 1.0508 & 2.5596 & 0.0140 \\
\hline
\end{tabular}


Table 6 List of gene ontologies (biological processes) significantly regulated by PFJ (Continued)

\begin{tabular}{lllll}
\hline GO:0019219 & $\begin{array}{l}\text { Regulation of nucleobase, nucleoside, nucleotide and nucleic } \\
\text { acid metabolic process }\end{array}$ & 21 & 0.9620 & 2.3612 \\
GO:0008219 & Cell death & 7 & 1.3514 & 2.0210 \\
\hline
\end{tabular}

signalling pathway was down-regulated in NRs given PFJ, including genes for mitogen-activated protein triple kinases, Map3k2 and Map3k11, phosphatidylinositol kinases, Pik3r3 and Pi4ka, as well as syntaxin binding protein $2(\operatorname{Stxbp} 2)$.

Insulin is essential for appropriate tissue development, growth and maintenance of whole body glucose homeostasis. This hormone is secreted by the $\beta$ cells of the pancreatic islets of Langerhans in response to increased circulating levels of glucose after a meal. Insulin regulates glucose homeostasis by reducing hepatic glucose output and increasing the rate of glucose uptake primarily into striated muscle and adipose tissues. In these tissues, the clearance of circulating glucose depends on the insulinstimulated translocation of the facilitative glucose transporter 4 (GLUT4) to the cell surface. Insulin also profoundly affects lipid metabolism by increasing lipid synthesis in liver and adipose tissues, as well as attenuating fatty acid release from TG in fat and muscle cells. Insulin resistance occurs when normal circulating concentrations of the hormone are insufficient to dispose of circulating glucose imposed by glucose-rich diets. In fact, insulin rises dramatically in concert with insulin resistance in the early diabetes of NRs fed high-glycaemic load diets, then falls as diabetes progresses [15].

To assure insulin sensitivity, the circulating hormone must bind to an enzyme that activates its functions, in this case the $\alpha$-subunit of the insulin receptor embedded in the cell membrane. This binding triggers the tyrosine kinase activity in the $\beta$-subunit of the insulin receptor, which further causes phosphorylation of two types of enzymes, mitogen-activated protein kinases (MAPKs) and phosphatidylinositol 3-kinases (PI3Ks), which are responsible for expressing the mitogenic and metabolic actions of insulin, respectively [111]. The activation of MAPKs leads to the completion of mitogenic functions such as cell growth and gene expression, while the activation of PI3Ks leads to important metabolic functions such as synthesis of lipids, proteins and glycogen, as well as cell survival and cell proliferation. Most importantly, the PI3K pathway is responsible for the distribution of glucose for essential cell functions.

\section{MAPKs}

In our present study, two enzymes involved in the MAPK pathway of insulin signalling, i.e. Map3k2 and Map3k11, were down-regulated in PFJ-supplemented rats. Many studies have causally implicated MAPKs in the development of insulin resistance [96]. Systemic insulin resistance triggers chronic hyperglycaemia, which causes pancreatic $\beta$ cells to secrete more insulin. In the long term, this adaptation is associated with stressinduced $\beta$ cell death and leads to insulin deficiency and T2DM. As such, stress mechanisms that trigger insulin resistance are also known to contribute to $\beta$ cell failure. The majority of studies indicate that prolonged enhanced MAPK signalling is detrimental to insulin sensitivity and $\beta$ cell function. A growing body of evidence also indicates that MAPKs are involved in physiological metabolic adaptation, the disturbance of which might contribute to metabolic diseases. Thus, although MAPK-dependent signal transduction is required for physiological metabolic adaptation, inappropriate MAPK signalling contributes to the development of T2DM and the metabolic syndrome [41].

\section{GLUT4}

By definition, insulin resistance is a defect in signal transduction associated with accumulation of diacylglycerol and ceramides [91, 101]. At present, only one class of downstream signalling molecules is confirmed to be essential for insulin-stimulated glucose uptake and GLUT4 translocation, i.e. the class IA PI3Ks [27]. The GLUT4 vesicle, which is responsible for passive diffusion of glucose, binds to PI3Ks after bringing glucose into the cell. PI3Ks isolate the GLUT4 vesicle from the glucose and send the vesicle back to the cell membrane. The glucose that is isolated is then sent to the mitochondria to produce energy as ATP, and excess glucose is stored in the cell as glycogen, which is increased in NRs with T2DM [21]. The binding of insulin to its receptor on the surface of adipose and muscle cells initiates a signalling cascade that alters the trafficking itinerary of GLUT4 thus releasing it from intracellular stores and delivering it to the cell surface $[18,109]$. In the absence of insulin, about $95 \%$ of GLUT4 is confined to intracellular compartments. Insulin stimulation results in GLUT4 redistribution from these intracellular stores to the plasma membrane via alterations in membrane trafficking [18, 109]. This insulin-stimulated translocation of GLUT4 from intracellular sites to the plasma membrane is defective in individuals with insulin resistance and T2DM thus providing an impetus to comprehend how this trafficking pathway is controlled $[12,44]$.

\section{PI3Ks}

Emerging data indicate that the products of class IA PI3Ks act as both membrane anchors and allosteric 
regulators, serving to localise and activate downstream enzymes and their protein substrates [106]. Several studies have suggested that the interaction of insulin receptor substrate (IRS) proteins with PI3Ks is necessary for the appropriate activation and/or targeting of the enzyme to a critical intracellular site, including its association with GLUT4 vesicles [91]. Class IA PI3Ks play an essential role in insulin stimulation of glucose transport and metabolism and protein and lipid synthesis, as well as cell growth and differentiation [98].

In terms of molecular structure, class IA PI3Ks are heterodimers consisting of one regulatory and one catalytic subunit, each of which occurs in multiple isoforms $[118,119]$. Three mammalian genes, Pik3r1, Pik3r2 and Pik3r3 encode for the $\mathrm{p} 85 \alpha$ (p85 $\alpha$, p $50 \alpha$ and $\mathrm{p} 55 \alpha$ isoforms), $\mathrm{p} 85 \beta$ and $\mathrm{p} 55 \gamma$ regulatory subunits, respectively. The family of the catalytic subunits includes $p 110 \alpha$, $\mathrm{p} 110 \beta$, and $\mathrm{p} 110 \delta$ [106]. These are the products of three respective genes, $P i k 3 c a, P i k 3 c b$ and $P i k 3 c d$. The regulatory subunits of class IA PI3Ks appear to play three important functional roles. They confer stability on the catalytic subunits, induce lipid kinase activity upon insulin stimulation [131] and, in the basal state, inhibit the catalytic activity of the p110 subunits to various degrees [116].

The unique structural domains of the PI3K regulatory subunits and their differential abundances in tissues suggest that they are not entirely redundant and may serve unique purposes. Complete disruption of hepatic Pik3r1 and Pik3r2 markedly reduces insulin-stimulated PI3K activity, at least in part by destabilising the catalytic subunits [112]. On the other hand, partial loss of the regulatory subunits of PI3Ks increases insulin sensitivity, and this appears to be related to diminished negative feedback to the IRS proteins [40]. For example, mice with a knockout of the full-length p85 $\alpha$ exhibit an upregulation of the splice variants $\mathrm{p} 50 \alpha$ and $\mathrm{p} 55 \alpha$ in muscle and fat tissues and have increased insulin sensitivity [114]. In addition, p50 $\alpha / \mathrm{p} 55 \alpha$ knockout mice exhibit improved insulin sensitivity, lower fat masses and protection against obesity-induced insulin resistance [23]. However, mice with complete deletion of p85 $\alpha$ and its short splice variants $\mathrm{p} 50 \alpha$ and $\mathrm{p} 55 \alpha$ die perinatally with liver necrosis and enlarged muscle fibres [38]. Thus, identifying the precise pathways uniquely mediated by these regulatory subunit isoforms remains an important area for further study.

In the present study, the Pik3r3 gene encoding for the p55y regulatory subunit of PI3Ks was down-regulated in NRs given PFJ. p55 $\gamma$ is similar in structure to $\mathrm{p} 55 \alpha$ but is expressed at low levels in most tissues [111]. However, the effect of inhibiting or knocking out p $55 \gamma$, encoded by the Pik3r3 gene, on insulin sensitivity has not been conclusively determined. Nevertheless, since rats given PFJ had lower levels of RBG $(p<0.05)$ but similar insulin levels compared to NRs in the control group, the downregulation of the Pik3r3 gene and the related hepatic insulin-signalling pathway in general suggests that reduced glucose absorption by PFJ lowered the diabetogenic effects of the high-carbohydrate diet and/or enhanced insulin sensitivity, rather than PFJ acting by increasing insulin secretion. This is in accordance with the physiological parameters, as outlined above. The down-regulation of the insulin-signalling pathway could prove beneficial in the long run, as this would protect the pancreas from overproducing insulin and preserve insulin sensitivity in the related target organs, thereby preventing hyperinsulinaemia and hyperglycaemia.

\section{Down-regulation of hepatic genes involved in fibrotic processes was observed in NRs given PFJ T2DM and hepatic diseases}

T2DM and obesity are risk factors for non-alcoholic fatty liver diseases, which include hepatic steatosis (nonalcoholic fatty liver disease or NAFLD), non-alcoholic steatohepatitis (NASH), fibrosis and cirrhosis. Increased insulin resistance and adiposity contribute to the progression from non-alcoholic steatohepatitis to fibrosis through the development of a pro-fibrotic condition in the liver, including increased hepatocellular death, increased generation of reactive oxygen species and an altered cytokine balance [24]. Liver disease is an important cause of death in T2DM, as T2DM is currently the most common cause of liver disease in the USA, including the hepatocellular carcinoma that results from chronic T2DM [115]. The prevalence of T2DM in cirrhosis is 12.3 to $57 \%$ [117].

Incidentally, hepatic steatosis is the most prevalent early lesions in diabetic NRs and is correlated with advancing T2DM, with hepatomegaly and liver discolouration also present macroscopically [70]. A large proportion of male NRs that reach 1 year of age with T2DM also reveal hepatocellular carcinoma in various stages (Kenneth C. Hayes, Brandeis University, MA, personal communication).

\section{Collagen accumulation and fibrosis}

Organ fibrosis including liver fibrosis is characterised by an excessive accumulation of collagen. Mature collagen cross-links in a variety of connective tissues such as bones, tendons, ligaments and cartilages are formed via the hydroxyallysine route. In contrast, collagen in the skin is mainly cross-linked via the allysine route. In organ fibrosis, an increase in cross-links derived from the hydroxyallysine route is found. This change in cross-linking is related to irreversible accumulation of collagen in fibrotic tissues. Collagen containing hydroxyallysine-derived cross-links is more difficult to degrade than collagen containing allysine-derived cross-links. Inhibition of the formation of hydroxyallysine- 


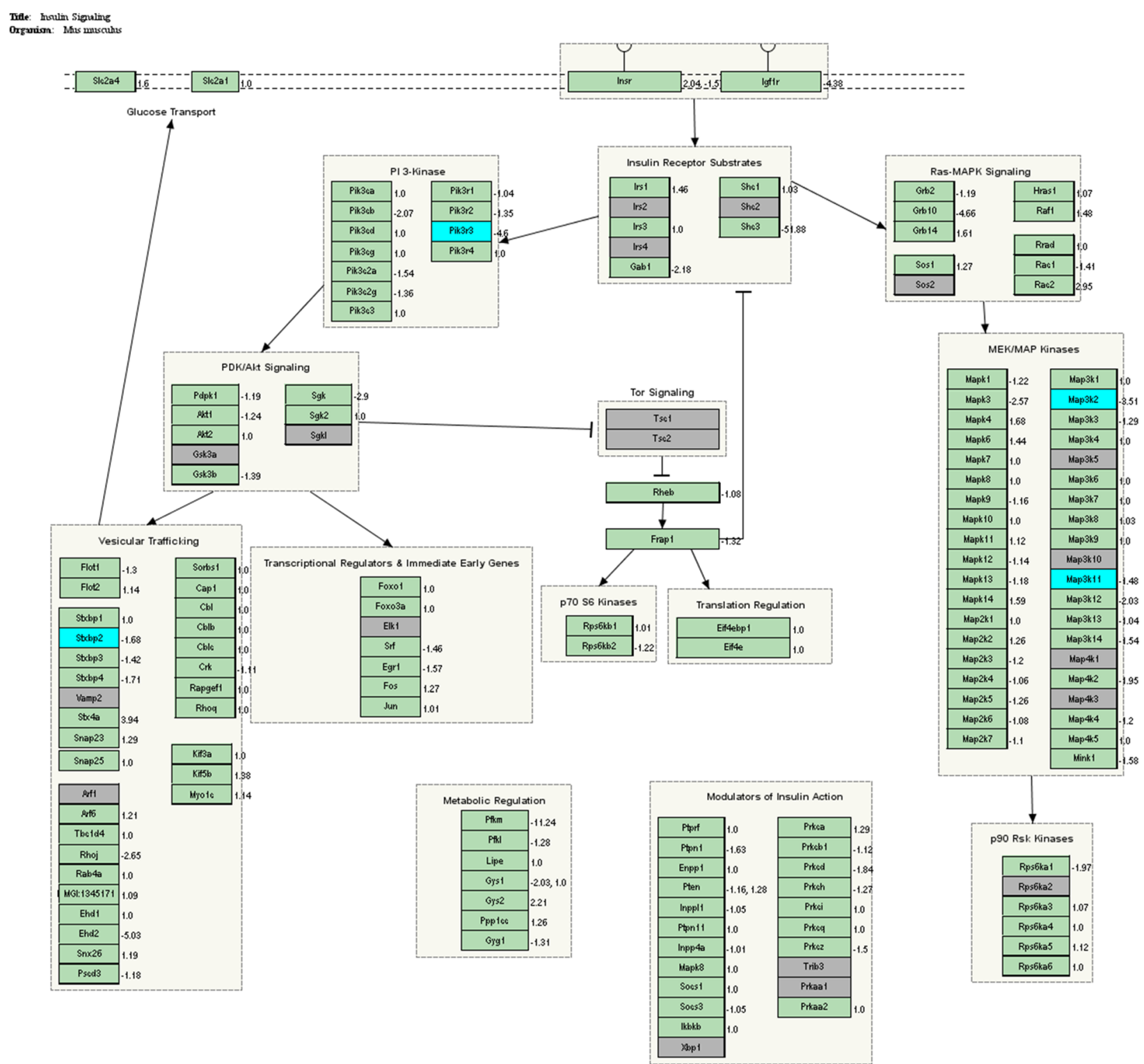

Fig. 1 Insulin-signalling pathway related genes down-regulated by PFJ in the liver of NRs

derived cross-links in fibrosis is therefore likely to result in the formation of collagen that is easier to degrade, thereby preventing unwanted collagen accumulation.

In the present study, two genes involved in fibrotic processes, i.e. Pcolce and Plod2, were found downregulated in the $\mathrm{PFJ}$ group. The procollagen $\mathrm{C}$ endopeptidase enhancer 1 (Pcolce) gene encodes a glycoprotein which binds and drives the enzymatic cleavage of type I procollagen and heightens C-proteinase activity, hence increasing fibrotic processes [108]. The increase in hydroxyallysine-derived cross-links in fibrosis is the result of an overhydroxylation of lysine residues within the collagen telopeptides, a function carried out by the enzyme encoded by procollagen-lysine, 2-oxoglutarate 5dioxygenase 2 (Plod2). Plod2 is thus involved in fibrotic processes as well [120].

\section{PFJ up-regulated hepatic apolipoprotein genes, especially} apolipoprotein $\mathbf{A} 1$

Metabolic pathways for the utilisation of carbohydrates and fats are intricately intertwined. In addition to having profound effects on carbohydrate metabolism, insulin also has important effects on lipid metabolism. One of these is to promote the synthesis of fatty acids in the liver when the organ is saturated with glycogen, and these fatty acids are then exported from the liver as lipoproteins, which are 


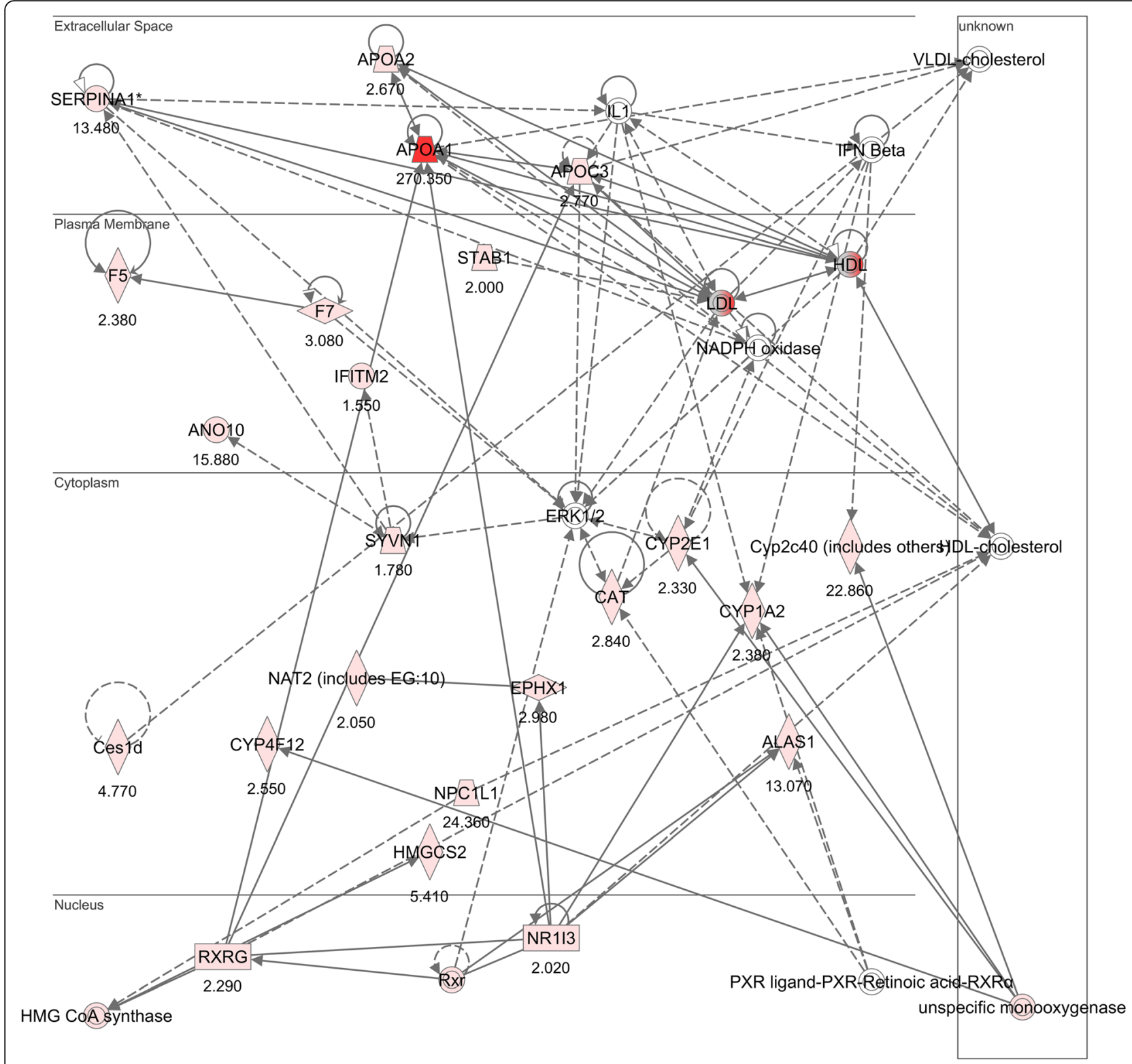

Fig. 2 Apolipoprotein genes up-regulated by PFJ in the liver of NRs

further catabolised in the circulation, eventually yielding free fatty acids for use by other tissues. Insulin resistance and T2DM are associated with plasma lipid and lipoprotein abnormalities, which include reduced high-density lipoproteins (HDL), a predominance of low-density lipoproteins (LDL) and elevated TG levels, also previously described in NRs with T2DM [14]. Increased hepatic secretion of very-low-density lipoproteins (VLDL) and their impaired clearance also appear to be of central importance in the pathophysiology of this diabetic dyslipaemia [62]. In T2DM, increased efflux of free fatty acids from adipose tissues and impaired insulin-mediated skeletal muscle uptake of free fatty acids also increase fatty acid flux to the liver $[11,59]$. Epidemiologic studies have demonstrated a relationship between insulin resistance and plasma free fatty acid levels [93]. In line with this, agents that lower elevated free fatty acids, such as thiazolidinediones, have been shown to improve insulin sensitivity in muscle, liver and adipose tissues [76, 78].

In the present study, genes up-regulated in the livers of NRs given PFJ include those encoding for apolipoproteins. The up-regulation of apolipoprotein genes, including Apoa1, Apoa2, Apoc1 and Apoc3, suggests an increase in HDL synthesis relative to controls, as all apolipoproteins A1, A2, C1 and C3 are components of HDL. The first step in HDL synthesis involves the secretion of apolipoprotein A1 mainly by the liver and the intestine [132, 133]. Apolipoproteins A1 and A2 are the 


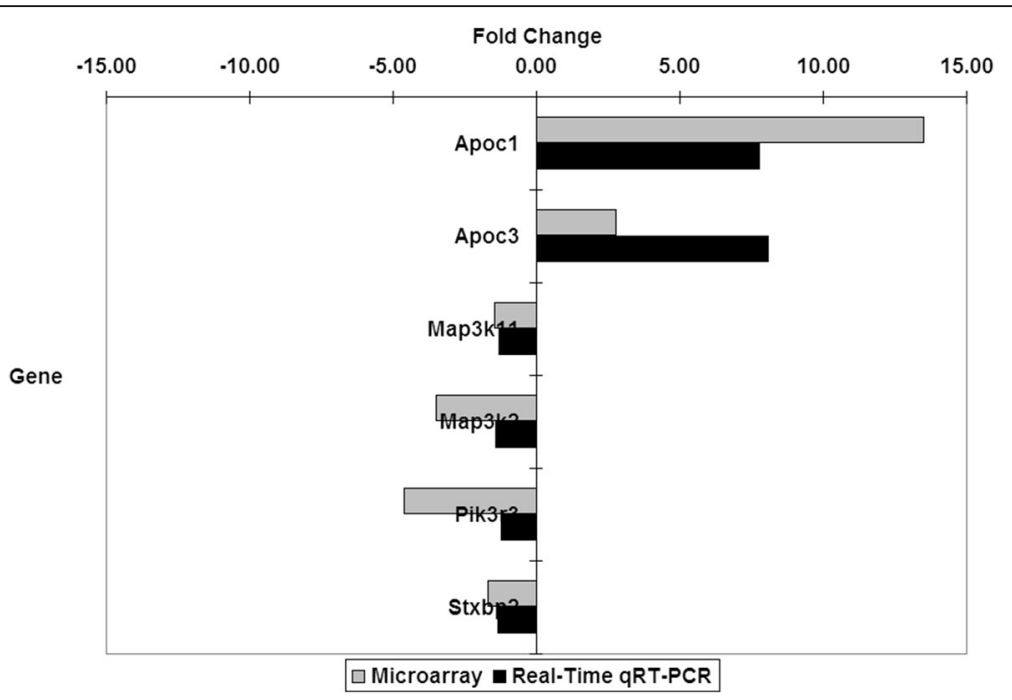

Fig. 3 Gene expression fold changes quantified by microarray and real-time qRT-PCR

main scaffold proteins that determine HDL particle structure [13]. Apolipoprotein A1 levels are reported to be inversely associated with diabetic retinopathy [51]. Apolipoproteins $\mathrm{C}$ are constituents of chylomicrons, VLDL and HDL [55]. However, in the fasting state, apolipoproteins $\mathrm{C}$ are mainly associated with HDL, whereas in the fed state, they preferentially redistribute to the surface of chylomicrons and VLDL [73]. Apolipoprotein $\mathrm{C} 1$ overexpression in transgenic mice has been associated with protection from obesity and insulin resistance [56]. On the contrary, apolipoprotein C3 deficiency has been reported to result in diet-induced obesity and aggravated insulin resistance in mice [31].

Virtually, every lipid and lipoprotein is affected by insulin resistance and T2DM, but the control of hyperglycaemia is unlikely to correct existing dyslipaemia. Although plasma glucose control is important in reducing microvascular complications due to T2DM, lipid management is also essential in these patients to decrease the incidence of cardiovascular events. In the present study, the up-regulation of apolipoproteins important in HDL synthesis appeared beneficial, as evidenced by the significantly lower amounts of plasma TG $(p<0.05)$ and adipose tissues $(p<0.05)$ in NRs given PFJ compared to the control group. Although we did not measure the levels of HDL in the present study, we have previously shown that PFJ increased plasma HDL levels of golden Syrian hamsters fed an atherogenic diet [6]. In line with this, green tea extract rich in phenolic compounds was also previously found to significantly reduce fasting TG and increase HDL in within-group analysis of people with T2DM, in addition to causing a decreasing trend of fasting TG in between-group analysis [69]. The increase in apolipoprotein A1 in these T2DM patients is also comparable with that in HDL after green tea extract supplementation [69].
Phase I and phase II detoxification genes were upregulated in the livers of NRs given PFJ

Phase I and phase II detoxification enzyme systems are involved in the degradation of xenobiotics. To some extent, phenolic compounds in general may be regarded as xenobiotics by animal cells and are treated as such through interactions with these enzymes [81]. Phase I detoxification in the liver involves the activation of a series of enzymes called the cytochrome P450 mixedfunction oxidases. These biotransformation enzymes function by oxidising, reducing or hydrolysing xenobiotics thus creating biotransformed intermediates [90]. Several cytochrome $\mathrm{P} 450$ genes involved in phase I detoxification, such as Cyp1a2, Cyp2c67, Cyp2e1 and Cyp4f14, were up-regulated in NRs given PFJ. This is consistent with our previous observations, whereby cytochrome P450 genes were also up-regulated in mice given PFJ [65]. Conversely, hepatic Cyp1a2 was found downregulated in diabetic and insulin resistant New Zealand obese mice [89], while a decrease in hepatic Cyp2e1 activity was reported in ob/ob mice and fa/fa Zucker rats [34]. Cyp4f14 plays a role in the inactivation of eicosanoids [60], which could be beneficial in reducing inflammation.

Phase II detoxification enzymes perform conjugation reactions such as acylation, acetylation, glucuronidation, methylation, sulfation and glutathione conjugation, which help to convert biotransformed intermediates into less toxic, water-soluble substances that are easily excreted or eliminated from the body [90]. Incidentally, three antioxidant genes involved in phase II detoxification, i.e. Ugt2b36, Cat and Gsto2, were up-regulated in the livers of NRs given PFJ. Ugt2b36 (uridine diphosphate glucuronosyltransferase 2 family, polypeptide B36) is a glycosyltransferase enzyme that catalyses the transfer 
of the glucuronic acid component of uridine diphosphate glucuronic acid to xenobiotics. Ugt2b36 messenger ribonucleic acid (mRNA) levels were found to decrease in aging mice [39]. Cat (catalase) is a very important enzyme which protects cells from oxidative damage, as it catalyses the decomposition of hydrogen peroxide to water and oxygen. Blood catalase activity in T2DM subjects was found decreased when compared to that in non-diabetic controls, and this consequently increased hydrogen peroxide in muscle cells [43]. Gsto2 (glutathione S-transferase omega-2) is an enzyme involved in glutathione conjugation. Patients with uncontrolled T2DM have severely deficient synthesis of glutathione attributed to limited precursor availability [104]. In addition, insulin administration is known to increase glutathione $\mathrm{S}$-transferase gene expression through the $\mathrm{PI} 3 \mathrm{~K} / \mathrm{AKT} / \mathrm{mTOR}$ pathway and decrease intracellular oxidative stress [36].

\section{Real-time qRT-PCR validated the microarray data obtained}

In the present study, the directions of fold changes of the target genes obtained from the real-time qRT-PCR technique as quantified by the qBase software [48] were comparable to those obtained from the microarray technique (Fig. 3). However, the magnitudes of fold changes obtained using real-time qRT-PCR were consistently lower than those obtained using microarrays. This has been described as the fold change compression phenomenon, which is caused by various technical microarray limitations, including limited dynamic range, signal saturations and cross hybridisations [127].

\section{Anti-diabetic effects of polyphenols and glucose homeostasis: does PFJ affect glucose absorption, insulin secretion or insulin sensitivity?}

In addition to improving insulin production and function, another approach to overcome T2DM is to reduce glucose absorption by inhibiting the activities of digestive enzymes for glucose release/production or those of enterocyte membrane transporters responsible for glucose transport. Phenolic compounds have been reported to influence the apparent glycaemic indices of foods and limit postprandial glucose increases through these mechanisms [129]. For instance, phenolic compounds from certain fruits have been shown to inhibit activities of $\alpha$-amylase and $\alpha$-glucosidase [77], and some even have the potential to replace or reduce the dose of acarbose required during clinical trials to improve postprandial glycaemic control in T2DM [10]. Enterocyte membrane transporters responsible for glucose absorption in the small intestine include sodiumdependent glucose transporter 1 (SGLT1) and glucose transporter 2 (GLUT2). SGLT1 is responsible for glucose entrance from the apical side of the intestinal lumen into enterocytes via active transport, while GLUT2 assists glucose exit from the basolateral side of the intestinal lumen into the hepatic portal vein via facilitated diffusion [102]. Phenolic compounds have also been shown to inhibit these two types of transporters in human intestinal Caco2 cell lines [54, 74].

We previously suggested that PFJ may slow the rate of glucose absorption, enhance insulin secretion and/or increase insulin sensitivity [16]. The results obtained in the present study indicate that the anti-diabetic effects of PFJ are likely due to mechanisms other than an increase in insulin secretion. This is because plasma insulin was not increased after PFJ supplementation in NRs, and another previous study also revealed that the early problem in NRs was insulin resistance with hyperinsulinaemia, not insulin insufficiency [15]. Nonetheless, it would be useful to conduct an insulin tolerance test on these NRs to further differentiate these two possible mechanisms.

\section{Insulin signalling in relation to longevity and chronic diseases: could the positive health effects of PFJ be attributed to modulation of insulin signalling?}

The insulin-signalling pathway is an evolutionarily conserved mechanism of longevity from yeast to humans [7]. Therefore, modulation of this pathway has been suggested as an avenue in extending longevity and battling chronic diseases. Ample genetic evidence demonstrates that mild inhibition of insulin-signalling components (including the insulin receptor, IRS proteins and PI3Ks) or overactivation of forkhead box protein $\mathrm{O}$ (FoxO) transcription factors contributes to lifespan extension with improved metabolic profiles [49, 113]. Interestingly, Ayyadevara et al. [3] reported that genetic disruption of insulin-like signalling extended lifespan in the nematode Caenorhabditis elegans and to a lesser degree in other taxa including fruit flies and mice. They found remarkable longevity and stress resistance of nematode PI3Knull mutants that lacked the PI3K catalytic subunit [3]. Interestingly, the PI3K pathway has paradoxically two opposite functions, i.e. impairment of its signalling activates FoxO factors and extends lifespan, whereas its overactivity triggers nuclear factor-kappa beta (NF- $\kappa \beta)$ signalling and accelerates the aging process. FoxO activation also causes concomitant enhancement of cellular stress resistance and protection, suppression of lowgrade inflammation and enhanced mitochondrial biogenesis [121]. NF- $k \beta$ signalling has been recognised as one of the targets of PI3K pathway. The NF- $\kappa \beta$ system is a pleiotropic factor regulating developmental processes, host defence systems and cellular survival functions [97]. Since the suppression of PI3K signalling can extend lifespan, this implies that excessive and sustained activation of PI3K signalling triggers the aging process. 
In addition, there is increasing evidence for an association between obesity, T2DM and cancer. Epidemiologic data suggest that insulin resistance with hyperinsulinaemia, as well as increased insulin and insulin-like growth factor-1 (IGF-1) signalling account for the relationship between these conditions. Besides influencing T2DM, the PI3K pathway itself is also implicated in cancer. PI3K signalling is activated in human cancers via several different mechanisms, including direct mutational activation or amplification of genes encoding key components of the PI3K pathway. Activation of the PI3K pathway results in the activation of protein kinase $B$ or AKT. AKT inhibits apoptosis and stimulates protein synthesis and cell proliferation. The fact that insulin receptor signalling can stimulate protein synthesis and inhibit apoptosis and the fact that IGF-1 receptor signalling enhances cell proliferation explain how hyperinsulinaemia and increased IGF-1 may result in tumour growth. These pathways thus represent an intricate balance, and disruption of this equilibrium may lead to obesity, T2DM and cancer. Uncontrolled signalling through the PI3K pathway also contributes to metastatic cancers [72]. Thus, understanding the intricacies of the PI3K pathway may provide new avenues in terms of extending longevity and overcoming chronic diseases [20].

It is thus exciting to find that PFJ down-regulated insulin signalling in the present study, as this pathway is a potential target for modulation of longevity and chronic diseases. It is also important to note that the Pik3r3 gene, down-regulated in the livers of NRs given PFJ in the present study, is considered an oncogene important for cell proliferation and tumour growth, as it is overexpressed in certain cancers [126]. It is also interesting, but not surprising, that the gene expression patterns with regards to insulin signalling observed in the present study were not found in previous hepatic transcriptomic analyses of BALB/c mice tested on a low-fat diet [65] (with the exception of up-regulated cytochrome P450 genes), given a high-fat atherogenic diet [67] or injected with myeloma cells [66], as mice are not predisposed to T2DM since they are HDL animals in general and do not easily develop the metabolic syndrome. Nevertheless, we have previously shown that PFJ displayed many beneficial effects on degenerative diseases in various animal models $[65-68,99,100,103]$. Therefore, from the results obtained in the present study, it would be noteworthy in future studies to investigate whether PFJ confers its positive effects on these diseases by modulating components of the insulin-signalling pathway, especially PI3Ks.

\section{Limitations of study}

We acknowledge that a limitation in the present study was that mouse (Mus musculus) microarrays and realtime qRT-PCR assays were used to assess the gene expression changes of the NR (Arvicanthis niloticus). However, the application of the NR as a laboratory diurnal rodent for biomedical research applicable to humans is relatively new [94]. Therefore, detailed knowledge of its physiology is still lacking, and its genome has not been sequenced. Accordingly, no commercial whole genome microarrays are currently available for this species. Nevertheless, cross hybridisation studies using microarrays have been conducted previously, such as studies involving hybridising monkey samples to human microarrays $[25,29,42,52,63,75]$. NRs belong to the Muridae family, as do mice and rats [124]. As with the standard laboratory rat, the NR is relatively insensitive to variations in photoperiod and does not hibernate. Compared to the standard laboratory rat however, the NR reaches asymptotic body mass early in life and does not show marked sexual dimorphism [94]. We have previously tried hybridising NR samples to rat (Rattus norvegicus) microarrays, but quality control of the hybridisation indicated that the hybridisation was not satisfactory (Vassilis Zannis, Boston University School of Medicine, MA, personal communication). On the other hand, the hybridisation of NR samples to mouse (Mus musculus) microarrays carried out in the present study was of high quality, enabling interpretation of the data obtained. Nevertheless, future studies to delve further into the transcriptomic effects of PFJ on NRs would benefit from the various next-generation sequencing technologies and platforms currently available. It would also be interesting to compare the effects of PFJ in different animal models, especially to identify whether species-specific genes are involved.

Another limitation in the present study was that microarray gene expression profiling was not carried out on pancreatic islet $\beta$ cells, the site for insulin production. Obtaining high-quality and intact RNA from the pancreatic $\beta$ cells is difficult, however, as the primary function of the pancreas is as an exocrine aid in digestion. The pancreas thus expresses large quantities of proteases, DNases and RNases that initiate an autolytic process almost immediately upon harvest [83]. In addition, some techniques also involve tedious pancreatic cannulation procedures and cause tissue artefacts. However, newer and simpler techniques are emerging, such as the perfusion method using RNase inhibitors [45] and modifications of standard phenol/guanidine thiocyanate lysis reagent protocols [4]. These emerging protocols could be used in future experiments to study the gene expression changes caused by PFJ in the pancreas.

\section{Conclusions}

Transcriptomic gene expression analysis using microarrays from the livers of young male NRs supplemented with PFJ to prevent T2DM induction showed that genes 
related to HDL apolipoproteins and hepatic detoxification were up-regulated, while genes related to insulin signalling and fibrosis were down-regulated. Based on the results obtained, it is more likely that the antidiabetic effects of PFJ may be due to mechanisms other than an increase in insulin secretion, as the levels of insulin were not increased after PFJ supplementation in NRs, and young NRs have high concentrations of insulin during diabetes induction that suggest insulin resistance is the primary defect [15]. Further studies to investigate whether PFJ confers its positive effects on degenerative diseases by modulating components of the insulinsignalling pathway are also warranted.

\section{Abbreviations}

ANOVA: Analysis of variance; cDNA: Complementary deoxyribonucleic acid; cRNA: Complementary ribonucleic acid; Ct: Threshold cycle; En: Energy; FBG: Fasting blood glucose; FoxO: Forkhead box protein O; GAE: Gallic acid equivalent; GLUT2: Glucose transporter 2; GLUT4: Glucose transporter 4; HDL: High-density lipoproteins; IGF-1: Insulin-like growth factor 1; IRS: Insulin receptor substrate; LDL: Low-density lipoproteins; MAPK: Mitogen-activated protein kinase; mRNA: Messenger ribonucleic acid; mTOR: Mammalian target of rapamycin; NF-kß: Nuclear factor-kappa beta; NR: Nile rat; NTC: Non-template control; PFJ: Palm fruit juice; PI3K: Phosphatidylinositol 3-kinase; qRTPCR: Quantitative reverse transcription-polymerase chain reaction; RBG: Random blood glucose; SD: Standard deviation; SGLT1: Sodium-dependent glucose transporter 1: T2DM: Type 2 diabetes mellitus: TC: Total cholesterol; TG: Triacylglycerol; VLDL: Very-low-density lipoproteins

\section{Acknowledgements}

The authors thank the Director-General of the Malaysian Palm Oil Board for the permission to publish this manuscript. They also thank the support staff of the Phenolics Group in the Malaysian Palm Oil Board for the preparation of PFJ. Fadi Chaabo from Brandeis University is also acknowledged for his assistance with the animal feeding experiments. The authors are also grateful to Karen Lai and Yulia Dushkina from Brandeis University for their technical assistance in the care and handling of the NR breeding colony.

\section{Availability of data and materials}

The datasets generated during the current study are available in the Gene Expression Omnibus repository, http://www.ncbi.nlm.nih.gov/geo/(Accession number: GSE64901).

\section{Funding}

This research was funded by the Malaysian Palm Oil Board and the Brandeis University Foster Biomedical Research Laboratory funds for research and teaching.

\section{Authors' contributions}

SSL carried out the gene expression experiments and analyses, interpreted the gene expression data and drafted the manuscript. JB carried out the animal feeding experiments, performed the animal sample collection and interpreted the animal data. AP performed statistical analyses on the physiological and biochemical parameters of the animal study. $\mathrm{KCH}$ designed the animal feeding and helped in the interpretation of the animal data. RS was involved in the preparation of PFJ and helped in the interpretation of the gene expression data. All authors participated in helpful discussions and read as well as approved the final manuscript.

\section{Competing interests}

The authors declare that they have no competing interests.

\section{Consent for publication}

Not applicable

\section{Ethics approval}

All experiments and procedures were approved by the Brandeis University Institutional Animal Care and Use Committee. All institutional and national guidelines for the care and use of laboratory animals were followed.

\section{Author details}

'Malaysian Palm Oil Board, No. 6, Persiaran Institusi, Bandar Baru Bangi, 43000 Kajang, Selangor, Malaysia. ${ }^{2}$ Brandeis University, 415 South Street, Waltham, MA 02454, USA.

Received: 2 August 2016 Accepted: 14 October 2016

Published online: 22 October 2016

\section{References}

1. Anhe FF, Desjardins Y, Pilon G, Dudonne S, Genovese MI, Lajolo FM, Marette A. Polyphenols and type 2 diabetes: a prospective review. Pharma Nutr. 2013;1:105-14.

2. Aprikian O, Duclos V, Guyot S, Besson C, Manach C, Bernalier A, Morand C, Remesy C, Demigne C. Apple pectin and a polyphenol-rich apple concentrate are more effective together than separately on cecal fermentations and plasma lipids in rats. J Nutr. 2003;133(6):1860-5.

3. Ayyadevara S, Alla R, Thaden JJ, Shmookler Reis RJ. Remarkable longevity and stress resistance of nematode PI3K-null mutants. Aging Cell. 2008;7(1):13-22.

4. Azevedo-Pouly AC, Elgamal OA, Schmittgen TD. RNA isolation from mouse pancreas: a ribonuclease-rich tissue. J Vis Exp. 2014;90:e51779.

5. Bahadoran Z, Mirmiran P, Azizi F. Dietary polyphenols as potential nutraceuticals in management of diabetes: a review. J Diabetes Metab Disord. 2013;12(1):43.

6. Balasundram N, Sundram K, Samman S. Phenolic-rich palm fruit juice raises plasma HDL-C concentrations and improves antioxidant status in Golden Syrian hamsters fed an atherogenic diet. Asia Pac J Clin Nutr. 2005; 14(Suppl):S75.

7. Barbieri M, Bonafe M, Franceschi C, Paolisso G. Insulin/IGF-I-signaling pathway: an evolutionarily conserved mechanism of longevity from yeast to humans. Am J Physiol Endocrinol Metab. 2003;285(5):E1064-1071.

8. Bauer F, Beulens JW, Van der AD, Wijmenga C, Grobbee DE, Spijkerman AM, Van der Schouw YT, Onland-Moret NC. Dietary patterns and the risk of type 2 diabetes in overweight and obese individuals. Eur J Nutr. 2013;52(3):1127-34.

9. Bhupathiraju SN, Tobias DK, Malik VS, Pan A, Hruby A, Manson JE, Willett WC, Hu FB. Glycemic index, glycemic load, and risk of type 2 diabetes: results from 3 large US cohorts and an updated meta-analysis. Am J Clin Nutr. 2014;100(1):218-32.

10. Boath AS, Stewart D, McDougall GJ. Berry components inhibit alphaglucosidase in vitro: synergies between acarbose and polyphenols from black currant and rowanberry. Food Chem. 2012;135(3):929-36.

11. Boden $\mathrm{G}$. Role of fatty acids in the pathogenesis of insulin resistance and NIDDM. Diabetes. 1997;46(1):3-10.

12. Bogan JS. Regulation of glucose transporter translocation in health and diabetes. Annu Rev Biochem. 2012:81:507-32.

13. Bolanos-Garcia VM, Miguel RN. On the structure and function of apolipoproteins: more than a family of lipid-binding proteins. Prog Biophys Mol Biol. 2003;83(1):47-68.

14. Bolsinger J, Pronczuk A, Hayes KC. Dietary carbohydrate dictates development of type 2 diabetes in the Nile rat. J Nutr Biochem. 2013;24(11):1945-52.

15. Bolsinger J, Pronczuk A, Landstrom M, Auerbach A, Hayes KC. Low glycemic load diets protect against metabolic syndrome and type 2 diabetes mellitus in the Nile rat. J Nutr Biochem. 2016, In press.

16. Bolsinger J, Pronczuk A, Sambanthamurthi R, Hayes KC. Anti-diabetic effects of palm fruit juice in the Nile rat (Arvicanthis niloticus). J Nutr Sci. 2014;3:e5.

17. Bray GA, Lovejoy JC, Smith SR, DeLany JP, Lefevre M, Hwang D, Ryan DH, York DA. The influence of different fats and fatty acids on obesity, insulin resistance and inflammation. J Nutr. 2002;132(9):2488-91.

18. Bryant NJ, Govers R, James DE. Regulated transport of the glucose transporter GLUT4. Nat Rev Mol Cell Biol. 2002;3(4):267-77.

19. Bustin SA, Nolan T. Pitfalls of quantitative real-time reverse-transcription polymerase chain reaction. J Biomol Tech. 2004;15(3):155-66.

20. Cantley LC. The phosphoinositide 3-kinase pathway. Science. 2002; 296(5573):1655-7.

21. Chaabo F, Pronczuk A, Maslova E, Hayes K. Nutritional correlates and dynamics of diabetes in the Nile rat (Arvicanthis niloticus): a novel model for diet-induced type 2 diabetes and the metabolic syndrome. Nutr Metab. 2010;7:29. 
22. Che Idris CA, Karupaiah T, Sundram K, Tan YA, Balasundram N, Leow SS, Nasruddin NS, Sambanthamurthi R. Oil palm phenolics and vitamin E reduce atherosclerosis in rabbits. J Funct Foods. 2014;7:541-50.

23. Chen D, Mauvais-Jarvis F, Bluher M, Fisher SJ, Jozsi A, Goodyear L, Ueki K, Kahn CR. p50alpha/p55alpha phosphoinositide 3-kinase knockout mice exhibit enhanced insulin sensitivity. Mol Cell Biol. 2004;24(1):320-9.

24. Chiang DJ, Pritchard MT, Nagy LE. Obesity, diabetes mellitus, and liver fibrosis. Am J Physiol Gastrointest Liver Physiol. 2011;300(5):G697-702.

25. Chismar JD, Mondala T, Fox HS, Roberts E, Langford D, Masliah E, Salomon DR, Head SR. Analysis of result variability from high-density oligonucleotide arrays comparing same-species and cross-species hybridizations. Biotechniques. 2002;33(3):516-8. 520, 522 passim.

26. Cuervo A, Valdes L, Salazar N, De los Reyes-Gavilan CG, Ruas-Madiedo P, Gueimonde M, Gonzalez S. Pilot study of diet and microbiota: interactive associations of fibers and polyphenols with human intestinal bacteria. J Agric Food Chem. 2014;62(23):5330-6.

27. Czech MP, Corvera S. Signaling mechanisms that regulate glucose transport. J Biol Chem. 1999;274(4):1865-8.

28. De Bock M, Derraik JG, Brennan CM, Biggs JB, Morgan PE, Hodgkinson SC, Hofman PL, Cutfield WS. Olive (Olea europaea L.) leaf polyphenols improve insulin sensitivity in middle-aged overweight men: a randomized, placebocontrolled, crossover trial. PLOS One. 2013;8(3):e57622.

29. Dillman 3rd JF, Phillips CS. Comparison of non-human primate and human whole blood tissue gene expression profiles. Toxicol Sci. 2005;87(1):306-14.

30. Duda-Chodak A, Tarko T, Satora P, Sroka P. Interaction of dietary compounds, especially polyphenols, with the intestinal microbiota: a review. Eur J Nutr. 2015;54(3):325-41.

31. Duivenvoorden I, Teusink B, Rensen PC, Romijn JA, Havekes LM, Voshol PJ. Apolipoprotein C3 deficiency results in diet-induced obesity and aggravated insulin resistance in mice. Diabetes. 2005;54(3):664-71.

32. Eckel RH, Alberti KG, Grundy SM, Zimmet PZ. The metabolic syndrome. Lancet. 2010;375(9710):181-3.

33. Edgar R, Domrachev M, Lash AE. Gene Expression Omnibus: NCBI gene expression and hybridization array data repository. Nucleic Acids Res. 2002;30(1):207-10.

34. Enriquez A, Leclercq I, Farrell GC, Robertson G. Altered expression of hepatic CYP2E1 and CYP4A in obese, diabetic ob/ob mice, and fa/fa Zucker rats. Biochem Biophys Res Commun. 1999;255(2):300-6.

35. Eshak ES, Iso H, Mizoue T, Inoue M, Noda M, Tsugane S. Soft drink, $100 \%$ fruit juice, and vegetable juice intakes and risk of diabetes mellitus. Clin Nutr. 2013;32(2):300-8.

36. Franco R, Schoneveld OJ, Pappa A, Panayiotidis MI. The central role of glutathione in the pathophysiology of human diseases. Arch Physiol Biochem. 2007;113(4-5):234-58.

37. Frejnagel S, Juskiewicz J. Dose-dependent effects of polyphenolic extracts from green tea, blue-berried honeysuckle, and chokeberry on rat caecal fermentation processes. Planta Med. 2011;77(9):888-93.

38. Fruman DA, Mauvais-Janvis F, Pollard DA, Yballe CM, Brazil D, Bronson RT, Kahn CR, Cantley LC. Hypoglycaemia, liver necrosis and perinatal death in mice lacking all isoforms of phosphoinositide 3-kinase p85 alpha. Nat Genet. 2000;26(3):379-82.

39. Fu ZD, Csanaky IL, Klaassen CD. Effects of aging on mRNA profiles for drug metabolizing enzymes and transporters in livers of male and female mice. Drug Metab Dispos. 2012;40(6):1216-25.

40. Geering B, Cutillas PR, Vanhaesebroeck B. Regulation of class IA PI3Ks: is there a role for monomeric PI3K subunits? Biochem Soc Trans. 2007:35(Pt 2):199-203.

41. Gehart $H$, Kumpf $S$, Ittner A, Ricci R. MAPK signalling in cellular metabolism: stress or wellness? EMBO Rep. 2010;11(11):834-40.

42. George MD, Sankaran S, Reay E, Gelli AC, Dandekar S. High-throughput gene expression profiling indicates dysregulation of intestinal cell cycle mediators and growth factors during primary simian immunodeficiency virus infection. Virology. 2003:312(1):84-94.

43. Goth L. Catalase deficiency and type 2 diabetes. Diabetes Care. 2008; 31(12):e93.

44. Graham TE, Kahn BB. Tissue-specific alterations of glucose transport and molecular mechanisms of intertissue communication in obesity and type 2 diabetes. Horm Metab Res. 2007;39(10):717-21.

45. Griffin M, Abu-El-Haija M, Abu-El-Haija M, Rokhlina T, Uc A. Simplified and versatile method for isolation of high-quality RNA from pancreas. Biotechniques. 2012;52(5):332-4.

46. Groop L, Pociot F. Genetics of diabetes-are we missing the genes or the disease? Mol Cell Endocrinol. 2014;382(1):726-39.
47. Hanhineva K, Torronen R, Bondia-Pons I, Pekkinen J, Kolehmainen M, Mykkanen $\mathrm{H}$, Poutanen K. Impact of dietary polyphenols on carbohydrate metabolism. Int J Mol Sci. 2010;11(4):1365-402.

48. Hellemans J, Mortier G, De Paepe A, Speleman F, Vandesompele J. qBase relative quantification framework and software for management and automated analysis of real-time quantitative PCR data. Genome Biol. 2007; 8(2):R19.

49. Holzenberger M, Kappeler L, De Magalhaes FC. IGF-1 signaling and aging. Exp Gerontol. 2004;39(11-12):1761-4.

50. Huang PL. A comprehensive definition for metabolic syndrome. Dis Model Mech. 2009;2(5-6):231-7.

51. Irshad M, Dubey R. Apolipoproteins and their role in different clinical conditions: an overview. Indian J Biochem Biophys. 2005;42(2):73-80.

52. Jacquelin B, Mayau V, Brysbaert G, Regnault B, Diop OM, Arenzana-Seisdedos F, Rogge L, Coppee JY, Barre-Sinoussi F, Benecke A, Muller-Trutwin MC. Long oligonucleotide microarrays for African green monkey gene expression profile analysis. FASEB J. 2007:21(12):3262-71.

53. Johnson IT. Anticarcinogenic effects of diet-related apoptosis in the colorectal mucosa. Food Chem Toxicol. 2002;40(8):1171-8.

54. Johnston K, Sharp P, Clifford M, Morgan L. Dietary polyphenols decrease glucose uptake by human intestinal Caco-2 cells. FEBS Lett. 2005:579(7):1653-7.

55. Jong MC, Hofker MH, Havekes LM. Role of ApoCs in lipoprotein metabolism: functional differences between ApoC1, ApoC2, and ApoC3. Arterioscler Thromb Vasc Biol. 1999;19(3):472-84

56. Jong MC, Voshol PJ, Muurling M, Dahlmans VE, Romijn JA, Pij H, Havekes LM. Protection from obesity and insulin resistance in mice overexpressing human apolipoprotein C1. Diabetes. 2001;50(12):2779-85.

57. Kaur J. A comprehensive review on metabolic syndrome. Cardiol Res Pract. 2014; 2014:943162

58. Kelder T, van lersel MP, Hanspers K, Kutmon M, Conklin BR, Evelo CT, Pico AR. WikiPathways: building research communities on biological pathways. Nucleic Acids Res. 2012;40(Database issue):D1301-1307.

59. Kelley DE, Simoneau JA. Impaired free fatty acid utilization by skeletal muscle in non-insulin-dependent diabetes mellitus. J Clin Invest. 1994;94(6):2349-56

60. Kikuta $Y$, Kasyu H, Kusunose E, Kusunose M. Expression and catalytic activity of mouse leukotriene B4 omega-hydroxylase, CYP4F14. Arch Biochem Biophys. 2000;383(2):225-32.

61. Kim HM, Kim J. The effects of green tea on obesity and type 2 diabetes. Diabetes Metab J. 2013:37(3):173-5.

62. Krauss RM. Lipids and lipoproteins in patients with type 2 diabetes. Diabetes Care. 2004;27(6):1496-504.

63. Lachance PE, Chaudhuri A. Microarray analysis of developmental plasticity in monkey primary visual cortex. J Neurochem. 2004;88(6):1455-69.

64. Lee C, Longo V (2016) Dietary restriction with and without caloric restriction for healthy aging. F1000Res 5.

65. Leow SS, Sekaran SD, Sundram K, Tan YA, Sambanthamurthi R. Differential transcriptomic profiles effected by oil palm phenolics indicate novel health outcomes. BMC Genomics. 2011:12:432.

66. Leow SS, Sekaran SD, Sundram K, Tan YA, Sambanthamurthi R. Gene expression changes in spleens and livers of tumour-bearing mice suggest delayed inflammation and attenuated cachexia in response to oil palm phenolics. J Nutrigenet Nutrigenomics. 2013a;6(6):305-326.

67. Leow SS, Sekaran SD, Sundram K, Tan YA, Sambanthamurthi R. Oil palm phenolics attenuate changes caused by an atherogenic diet in mice. Eur $J$ Nutr. 2013b;52(2):443-456.

68. Leow SS, Sekaran SD, Tan YA, Sundram K, Sambanthamurthi R. Oil palm phenolics confer neuroprotective effects involving cognitive and motor functions in mice. Nutr Neurosci. 2013c;16(5):207-217.

69. Liu CY, Huang CJ, Huang LH, Chen IJ, Chiu JP, Hsu CH. Effects of green tea extract on insulin resistance and glucagon-like peptide 1 in patients with type 2 diabetes and lipid abnormalities: a randomized, double-blinded, and placebo-controlled trial. PLoS One. 2014;9(3), e91163.

70. Lyons J, Brown F, Remillard DE, Bolsinger J, Hayes KC. Pathology of the Nile rat developing type 2 diabetes [abstract]. Faseb J. 2013;27(Meeting Abstract Supplement):874.813.

71. Lyssenko V, Jonsson A, Almgren P, Pulizzi N, Isomaa B, Tuomi T, Berglund G, Altshuler D, Nilsson P, Groop L. Clinical risk factors, DNA variants, and the development of type 2 diabetes. N Engl J Med. 2008;359(21):2220-32.

72. Maehama T, Dixon JE. PTEN: a tumour suppressor that functions as a phospholipid phosphatase. Trends Cell Biol. 1999;9(4):125-8. 
73. Mahley RW, Innerarity TL, Rall Jr SC, Weisgraber KH. Plasma lipoproteins: apolipoprotein structure and function. J Lipid Res. 1984;25(12):1277-94.

74. Manzano S, Williamson G. Polyphenols and phenolic acids from strawberry and apple decrease glucose uptake and transport by human intestinal Caco-2 cells. Mol Nutr Food Res. 2010;54(12):1773-80.

75. Marvanova M, Menager J, Bezard E, Bontrop RE, Pradier L, Wong G. Microarray analysis of nonhuman primates: validation of experimental models in neurological disorders. FASEB J. 2003;17(8):929-31.

76. Mayerson AB, Hundal RS, Dufour S, Lebon V, Befroy D, Cline GW, Enocksson S, Inzucchi SE, Shulman Gl, Petersen KF. The effects of rosiglitazone on insulin sensitivity, lipolysis, and hepatic and skeletal muscle triglyceride content in patients with type 2 diabetes. Diabetes. 2002;51(3):797-802

77. McDougall GJ, Shpiro F, Dobson P, Smith P, Blake A, Stewart D. Different polyphenolic components of soft fruits inhibit alpha-amylase and alphaglucosidase. J Agric Food Chem. 2005;53(7):2760-6.

78. Miyazaki Y, Mahankali A, Matsuda M, Mahankali S, Hardies J, Cusi K, Mandarino LJ, DeFronzo RA. Effect of pioglitazone on abdominal fat distribution and insulin sensitivity in type 2 diabetic patients. J Clin Endocrinol Metab. 2002;87(6):2784-91.

79. Moco S, Martin FP, Rezzi S. Metabolomics view on gut microbiome modulation by polyphenol-rich foods. J Proteome Res. 2012;11(10):4781-90.

80. Morimoto A, Ohno Y, Tatsumi Y, Mizuno S, Watanabe S. Effects of healthy dietary pattern and other lifestyle factors on incidence of diabetes in a rural Japanese population. Asia Pac J Clin Nutr. 2012;21(4):601-8.

81. Moskaug JO, Carlsen H, Myhrstad MC, Blomhoff R. Polyphenols and glutathione synthesis regulation. Am J Clin Nutr. 2005:81(1 Suppl):277S-83S.

82. Movahed A, Nabipour I, Lieben Louis X, Thandapilly SJ, Yu L, Kalantarhormozi M, Rekabpour SJ, Netticadan T. Antihyperglycemic effects of short term resveratrol supplementation in type 2 diabetic patients. Evid Based Complement Alternat Med. 2013;2013:851267.

83. Mullin AE, Soukatcheva G, Verchere CB, Chantler JK. Application of in situ ductal perfusion to facilitate isolation of high-quality RNA from mouse pancreas. Biotechniques. 2006;40(5):617-21.

84. Neyrinck AM, Van Hee VF, Bindels LB, De Backer F, Cani PD, Delzenne NM. Polyphenol-rich extract of pomegranate peel alleviates tissue inflammation and hypercholesterolaemia in high-fat diet-induced obese mice: potential implication of the gut microbiota. Br J Nutr. 2013;109(5):802-9.

85. Noda K, Melhorn MI, Zandi S, Frimmel S, Tayyari F, Hisatomi T, Almulki L, Pronczuk A, Hayes KC, Hafezi-Moghadam A. An animal model of spontaneous metabolic syndrome: Nile grass rat. Faseb J. 2010;24(7):2443-53.

86. Nolan T, Hands RE, Bustin SA. Quantification of mRNA using real-time RTPCR. Nat Protoc. 2006;1(3):1559-82.

87. Olokoba AB, Obateru OA, Olokoba LB. Type 2 diabetes mellitus: a review of current trends. Oman Med J. 2012;27(4):269-73.

88. Osman HF, Eshak MG, El-Sherbiny EM, Bayoumi MM. Biochemical and genetical evaluation of pomegranate impact on diabetes mellitus induced by alloxan in female rats. Life Sci J. 2012;9(3):1543-53.

89. Pass GJ, Becker W, Kluge R, Linnartz K, Plum L, Giesen K, Joost HG. Effect of hyperinsulinemia and type 2 diabetes-like hyperglycemia on expression of hepatic cytochrome p450 and glutathione s-transferase isoforms in a New Zealand obese-derived mouse backcross population. J Pharmacol Exp Ther. 2002;302(2):442-50.

90. Percival M. Phytonutrients and detoxification. Clin Nutr Insights. 1997:5(2):1-4.

91. Pessin JE, Saltiel AR. Signaling pathways in insulin action: molecular targets of insulin resistance. J Clin Invest. 2000;106(2):165-9.

92. Pico AR, Kelder T, van lersel MP, Hanspers K, Conklin BR, Evelo C. WikiPathways: pathway editing for the people. PLoS Biol. 2008;6(7):e184.

93. Reaven GM, Chen YD. Role of abnormal free fatty acid metabolism in the development of non-insulin-dependent diabetes mellitus. Am J Med. 1988; 85(5A):106-12.

94. Refinetti R. The Nile grass rat as a laboratory animal. Lab Anim (NY). 2004; 33(9):54-7.

95. Romo-Vaquero M, Selma MV, Larrosa M, Obiol M, Garcia-Villalba R, GonzalezBarrio R, Issaly N, Flanagan J, Roller M, Tomas-Barberan FA, Garcia-Conesa MT. A rosemary extract rich in carnosic acid selectively modulates caecum microbiota and inhibits beta-glucosidase activity, altering fiber and short chain fatty acids fecal excretion in lean and obese female rats. PLoS One. 2014;9(4):e94687.

96. Sabio G, Davis RJ. cJun NH2-terminal kinase 1 (JNK1): roles in metabolic regulation of insulin resistance. Trends Biochem Sci. 2010;35(9):490-6.
97. Salminen A, Kaarniranta K. Insulin/IGF-1 paradox of aging: regulation via AKT/IKK/NF-kappaB signaling. Cell Signal. 2010;22(4):573-7.

98. Saltiel $A R$, Kahn CR. Insulin signalling and the regulation of glucose and lipid metabolism. Nature. 2001:414(6865):799-806.

99. Sambanthamurthi R, Tan YA, Sundram K, Abeywardena M, Sambandan TG, Rha C, Sinskey AJ, Subramaniam K, Leow SS, Hayes KC, Wahid MB. Oil palm vegetation liquor: a new source of phenolic bioactives. Br J Nutr. 2011a; 106(11):1655-1663.

100. Sambanthamurthi R, Tan YA, Sundram K, Hayes KC, Abeywardena M, Leow SS, Sekaran SD, Sambandan TG, Rha C, Sinskey AJ, Subramaniam K, Fairus S, Wahid MB. Positive outcomes of oil palm phenolics on degenerative diseases in animal models. Br J Nutr. 2011b;106(11):1664-1675.

101. Samuel VT, Shulman Gl. Mechanisms for insulin resistance: common threads and missing links. Cell. 2012;148(5):852-71.

102. Scheepers A, Joost HG, Schurmann A. The glucose transporter families SGLT and GLUT: molecular basis of normal and aberrant function. JPEN J Parenter Enteral Nutr. 2004;28(5):364-71.

103. Sekaran SD, Leow SS, Abobaker N, Tee KK, Sundram K, Sambanthamurthi R, Wahid MB. Effects of oil palm phenolics on tumor cells in vitro and in vivo. Afr J Food Sci. 2010;4(8):495-502.

104. Sekhar RV, McKay SV, Patel SG, Guthikonda AP, Reddy VT, Balasubramanyam A, Jahoor F. Glutathione synthesis is diminished in patients with uncontrolled diabetes and restored by dietary supplementation with cysteine and glycine. Diabetes Care. 2011;34(1):162-7.

105. Shen J, Goyal A, Sperling L. The emerging epidemic of obesity, diabetes, and the metabolic syndrome in china. Cardiol Res Pract. 2012;2012:178675.

106. Shepherd PR, Withers DJ, Siddle K. Phosphoinositide 3-kinase: the key switch mechanism in insulin signalling. Biochem J. 1998;333(Pt 3):471-90.

107. Slavin J. Fiber and prebiotics: mechanisms and health benefits. Nutrients. 2013;5(4):1417-35.

108. Steiglitz BM, Kreider JM, Frankenburg EP, Pappano WN, Hoffman GG, Meganck JA, Liang X, Hook M, Birk DE, Goldstein SA, Greenspan DS. Procollagen $C$ proteinase enhancer 1 genes are important determinants of the mechanical properties and geometry of bone and the ultrastructure of connective tissues. Mol Cell Biol. 2006;26(1):238-49.

109. Stockli J, Fazakerley DJ, James DE. GLUT4 exocytosis. J Cell Sci. 2011;124(Pt 24):4147-59.

110. Szkudelski T, Szkudelska K. Anti-diabetic effects of resveratrol. Ann N Y Acad Sci. 2011;1215:34-9.

111. Taniguchi CM, Emanuelli B, Kahn CR. Critical nodes in signalling pathways: insights into insulin action. Nat Rev Mol Cell Biol. 2006a;7(2):85-96.

112. Taniguchi CM, Kondo T, Sajan M, Luo J, Bronson R, Asano T, Farese R, Cantley LC, Kahn CR. Divergent regulation of hepatic glucose and lipid metabolism by phosphoinositide 3-kinase via Akt and PKClambda/zeta. Cell Metab. 2006b;3(5):343-353.

113. Tatar $M$, Bartke $A$, Antebi A. The endocrine regulation of aging by insulinlike signals. Science. 2003;299(5611):1346-51.

114. Terauchi Y, Tsuji Y, Satoh S, Minoura H, Murakami K, Okuno A, Inukai K, Asano T, Kaburagi Y, Ueki K, Nakajima H, Hanafusa T, Matsuzawa Y, Sekihara H, Yin Y, Barrett JC, Oda H, Ishikawa T, Akanuma Y, Komuro I, Suzuki M, Yamamura K, Kodama T, Suzuki H, Yamamura K, Kodama T, Suzuki H, Koyasu S, Aizawa S, Tobe K, Fukui Y, Yazaki Y, Kadowaki T. Increased insulin sensitivity and hypoglycaemia in mice lacking the p85 alpha subunit of phosphoinositide 3-kinase. Nat Genet. 1999;21(2):230-5.

115. Tolman KG, Fonseca $V$, Dalpiaz A, Tan MH. Spectrum of liver disease in type 2 diabetes and management of patients with diabetes and liver disease. Diabetes Care. 2007;30(3):734-43.

116. Tozzo E, Gnudi L, Kahn BB. Amelioration of insulin resistance in streptozotocin diabetic mice by transgenic overexpression of GLUT4 driven by an adipose-specific promoter. Endocrinology. 1997;138(4):1604-11.

117. Trombetta M, Spiazzi G, Zoppini G, Muggeo M. Review article: type 2 diabetes and chronic liver disease in the Verona diabetes study. Aliment Pharmacol Ther. 2005;22 Suppl 2:24-7.

118. Ueki K, Algenstaedt P, Mauvais-Jarvis F, Kahn CR. Positive and negative regulation of phosphoinositide 3-kinase-dependent signaling pathways by three different gene products of the p85alpha regulatory subunit. Mol Cell Biol. 2000;20(21):8035-46.

119. Ueki K, Fruman DA, Yballe CM, Fasshauer M, Klein J, Asano T, Cantley LC, Kahn CR. Positive and negative roles of p85 alpha and p85 beta regulatory subunits of phosphoinositide 3-kinase in insulin signaling. J Biol Chem. 2003;278(48):48453-66. 
120. van der Slot AJ, Zuurmond AM, Bardoel AF, Wijmenga C, Pruijs HE, Sillence DO, Brinckmann J, Abraham DJ, Black CM, Verzij N, DeGroot J, Hanemaaijer R, TeKoppele JM, Huizinga TW, Bank RA. Identification of PLOD2 as telopeptide lysyl hydroxylase, an important enzyme in fibrosis. J Biol Chem. 2003;278(42):40967-72.

121. van Heemst D. Insulin, IGF-1 and longevity. Aging Dis. 2010;1(2):147-57.

122. van lersel MP, Kelder T, Pico AR, Hanspers K, Coort S, Conklin BR, Evelo C. Presenting and exploring biological pathways with PathVisio. BMC Bioinformatics. 2008;9:399.

123. Vandesompele J, De Preter K, Pattyn F, Poppe B, Van Roy N, De Paepe A, Speleman F. Accurate normalization of real-time quantitative RT-PCR data by geometric averaging of multiple internal control genes. Genome Biol. 2002;3(7):RESEARCH0034

124. Volobouev VT, Ducroz JF, Aniskin VM, Britton-Davidian J, Castiglia R, Dobigny G, Granjon L, Lombard M, Corti M, Sicard B, Capanna E. Chromosomal characterization of Arvicanthis species (Rodentia, Murinae) from western and central Africa: implications for taxonomy. Cytogenet Genome Res. 2002;96(1-4):250-60.

125. Wainstein J, Ganz T, Boaz M, Bar Dayan Y, Dolev E, Kerem Z, Madar Z. Olive leaf extract as a hypoglycemic agent in both human diabetic subjects and in rats. J Med Food. 2012;15(7):605-10.

126. Wang G, Yang X, Li C, Cao X, Luo X, Hu J. PIK3R3 induces epithelial-tomesenchymal transition and promotes metastasis in colorectal cancer. Mol Cancer Ther. 2014;13(7):1837-47.

127. Wang Y, Barbacioru C, Hyland F, Xiao W, Hunkapiller KL, Blake J, Chan F, Gonzalez C, Zhang L, Samaha RR. Large scale real-time PCR validation on gene expression measurements from two commercial long-oligonucleotide microarrays. BMC Genomics. 2006;7:59.

128. Wang YW, Sun GD, Sun J, Liu SJ, Wang J, Xu XH, Miao LN. Spontaneous type 2 diabetic rodent models. J Diab Res. 2013;2013:401723.

129. Williamson G. Possible effects of dietary polyphenols on sugar absorption and digestion. Mol Nutr Food Res. 2013:57(1):48-57.

130. Wu GD, Chen J, Hoffmann C, Bittinger K, Chen YY, Keilbaugh SA, Bewtra M, Knights D, Walters WA, Knight R, Sinha R, Gilroy E, Gupta K, Baldassano R, Nessel L, Li H, Bushman FD, Lewis JD. Linking long-term dietary patterns with gut microbial enterotypes. Science. 2011;334(6052):105-8.

131. Yu J, Zhang Y, Mcllroy J, Rordorf-Nikolic T, Orr GA, Backer JM. Regulation of the p85/p110 phosphatidylinositol $3^{\prime}$-kinase: stabilization and inhibition of the p110alpha catalytic subunit by the p85 regulatory subunit. Mol Cell Biol. 1998;18(3):1379-87.

132. Zannis VI, Cole FS, Jackson CL, Kurnit DM, Karathanasis SK. Distribution of apolipoprotein A-I, C-II, C-III, and E mRNA in fetal human tissues. Timedependent induction of apolipoprotein E mRNA by cultures of human monocyte-macrophages. Biochemistry. 1985;24(16):4450-5.

133. Zannis VI, Fotakis P, Koukos G, Kardassis D, Ehnholm C, Jauhiainen M, Chroni A. HDL biogenesis, remodeling, and catabolism. Handb Exp Pharmacol. 2015;224:53-111.

\section{Submit your next manuscript to BioMed Central and we will help you at every step:}

- We accept pre-submission inquiries

- Our selector tool helps you to find the most relevant journal

- We provide round the clock customer support

- Convenient online submission

- Thorough peer review

- Inclusion in PubMed and all major indexing services

- Maximum visibility for your research

Submit your manuscript at www.biomedcentral.com/submit

) Biomed Central 